\title{
Studies on Cerebral Protective Agents. X. Synthesis and Evaluation of Anticonvulsant Activities for Novel 4,5,6,7-Tetrahydrothieno[3,2-c]pyridines and Related Compounds ${ }^{1)}$
}

\author{
Mitsuru OhKubo, ${ }^{*, a}$ Atsushi Kuno, ${ }^{a}$ Kiyotaka Katsuta, ${ }^{a}$ Yoshiko Ueda, ${ }^{a}$ \\ Kiyoharu Shirakawa, ${ }^{a}$ Hajime NaKanishi, ${ }^{a}$ Takayoshi Kinoshita, ${ }^{b}$ and \\ Hisashi TAKASUGI ${ }^{a}$ \\ New Drug Research Laboratories ${ }^{a}$ and Analytical Research Laboratories, ${ }^{b}$ Fujisawa Pharmaceutical Co., Ltd., 1-6 \\ Kashima 2-chome, Yodogawa-ku, Osaka 532, Japan. Received October 11, 1995; accepted December 9, 1995
}

\begin{abstract}
Novel 4,5,6,7-tetrahydrothieno[3,2-c]pyridines, 1-thienyl-1,2,3,4-tetrahydroisoquinolines and related compounds, in which the benzene rings of $(+)-1$ (FR115427) were replaced with heteroaromatic rings such as thiophene, furan, benzothiophene and indole, were synthesized and evaluated for anticonvulsant activity against i.c.v. $N$-methyl-D-aspartate (NMDA)-induced seizures in mice. Among these compounds, (+)-4-methyl-4-phenyl-4,5,6,7-

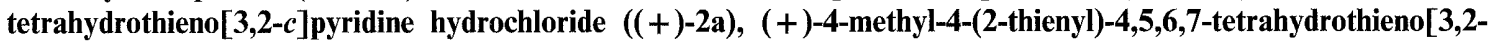
c]pyridine hydrochloride $((+)-2 \mathrm{~g})$, and (-)-1-methyl-1-(2-thienyl)-1,2,3,4-tetrahydroisoquinoline hydrochloride $((-)-3 a)$ showed significant anticonvulsant activity. The structure-activity relationships with regard to the anticonvulsant activity of this series of compounds are discussed.
\end{abstract}

Key words tetrahydrothieno[3,2-c]pyridine; tetrahydroisoquinoline; $N$-methyl-D-aspartate; anticonvulsant; structure activity relationship; FR 115427

In a previous paper, ${ }^{1)}$ we reported that $(+)-(S)-1-$ methyl-1-phenyl-1,2,3,4-tetrahydroisoquinoline hydrochloride ((+)-1; FR115427) (Fig. 1), a non-competitive $N$-methyl-D-aspartate (NMDA) antagonist, exhibited anticonvulsant activity against i.c.v. NMDA-induced seizures and cerebral protective activities in animal models.

A thiophene ring is known to be an appropriate bioisosteric replacement for a benzene ring. In fact, $N$ [1-(2-thienyl)cyclohexan-1-yl]piperidine (TCP), the thiophene analog of phencyclidine (PCP), also showed anticonvulsant activity against NMDA-induced seizures. ${ }^{2)}$ In order to investigate the influence of structural changes, we decided to replace the benzene rings of FR115427 with heteroaromatic rings (e.g. thiophene, furan, benzothiophene and indole). Thus, the thiophene derivatives $(\mathbf{2}, \mathbf{3})$ (Fig. 1) and related compounds were synthesized and evaluated for in vivo NMDA-antagonist activity. This was assessed by evaluation of anticonvulsant activity against i.c.v. NMDA-induced seizures in mice. In this paper, we describe the synthesis and the structure-activity relationships (SAR) with regard to anticonvulsant activity of these compounds.

\section{Chemistry}

Synthesis of 4,5,6,7-tetrahydrothieno[3,2-c]pyridine (2a) was performed by means of the four methods described in Charts $1-3$. The starting compound 6,7dihydrothieno $[3,2-c]$ pyridine $(\mathbf{5 a})^{3)}$ was prepared from $N$-benzoyl-2-(2-aminoethyl)thiophene via the BischlerNapieralski reaction. The yields of $\mathbf{2 a}$ from $\mathbf{5 a}$ in these methods are shown in Table 1.

The route used in method A (Chart 1) has already been reported in the previous paper. ${ }^{1}{ }^{1}$ In the case of sulfur-containing compounds such as $\mathbf{2 a}$, however, the benzyl group of 7a was removed by catalytic hydrogenation to afford $2 \mathrm{a}$ in only $6.0 \%$ yield. Alternatively, oxidative removal of the methoxybenzyl group of $\mathbf{7 b}$ (method B, Chart 1) was achieved using ceric ammonium nitrate (CAN) to afford $\mathbf{2 a}$ in $19.7 \%$ yield.

Direct introduction of the methyl group is illustrated in Chart 2 (method C). Activation of reactivity at the C-4 position of the 6,7-dihydrothieno[3,2-c]pyridine ring with a Lewis acid, such as boron trifluoride, was followed by alkylation with alkyllithium to afford 2a in $15.2 \%$ yield by one step from $\mathbf{5 a}$.

Another method via the nitrone intermediate (8a) is shown in Chart 3 (method D). Alkylation at the C-1 position of the nitrone (8a), which was prepared by oxidation of $\mathbf{5 a}$ with $m$-chloroperbenzoic acid, was achieved by means of the Grignard reaction to afford the hydroxylamine (9a). Reduction of $9 \mathbf{a}$ with phosphorus trichloride $\left(\mathrm{PCl}_{3}\right)$ afforded $2 \mathrm{a}$ in $27.9 \%$ yield from $5 \mathbf{a}$. This route was good in terms of total yield from 5a, although there were four steps (Table 1). The related racemic compounds $\mathbf{2 b}-\mathbf{4 c}$ were synthesized by use of the methods given in Table 2. Optical resolution of $\mathbf{2 a}, \mathbf{g}$ and 3a was performed using (+)- and (-)-di-p-toluoyl-Dtartaric acids as the resolving agents.

\section{Pharmacological Results and Discussion}

The compounds listed in Table 2 were tested for anticonvulsant activity in mice against i.c.v. NMDA $(0.32 \mu \mathrm{g})$-induced seizures as described previously. ${ }^{1)}$

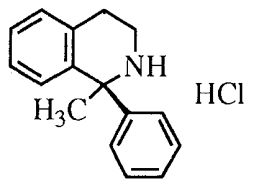

$(+)-1$

(FR1 15427)

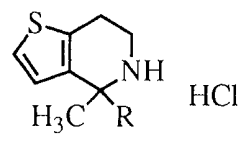

$$
\begin{aligned}
& \text { 2a: } R=P h \\
& \text { 2g: } R=2 \text {-thienyl }
\end{aligned}
$$

Fig. 1<smiles>CC1(c2cccs2)NCCc2ccccc21</smiles>

$3 a$
(C) 1996 Pharmaceutical Society of Japan 


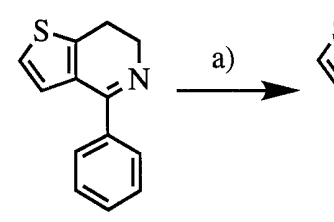

$5 a$<smiles>[X]c1ccc(C[N+]2=C(c3ccccc3)c3ccsc3CC2)cc1</smiles>

6a; $\mathrm{X}=\mathrm{H}$ $\mathbf{6 b} ; \mathrm{X}=\mathrm{OCH}_{3}$

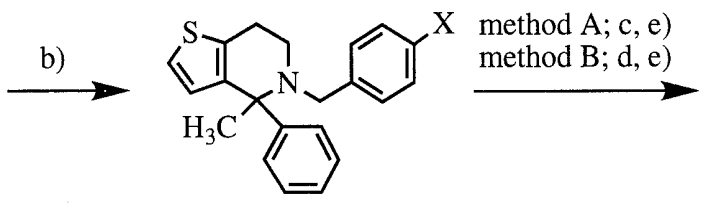

$7 \mathbf{a} ; \mathrm{X}=\mathrm{H}$ $7 \mathbf{b} ; \mathrm{X}=\mathrm{OCH}_{3}$<smiles>CC1(c2ccccc2)NCCc2sccc21</smiles>

$2 \mathbf{a}$

a) $\mathrm{ArCH}_{2} \mathrm{I} / \mathrm{CH}_{3} \mathrm{CN}$; b) $\mathrm{CH}_{3} \mathrm{MgBr} / \mathrm{THF}$; c) $\mathrm{H}_{2}, 10 \% \mathrm{Pd}-\mathrm{C} / \mathrm{CH}_{3} \mathrm{COOH}$; d) $\left(\mathrm{NH}_{4}\right)_{2} \mathrm{Ce}\left(\mathrm{NO}_{3}\right)_{6} / \mathrm{CH}_{3} \mathrm{CN}-\mathrm{H}_{2} \mathrm{O}$; e) $\mathrm{HCl} / \mathrm{EtOH}$

Chart 1

Method C<smiles>c1ccc(C2=NCCc3sccc32)cc1</smiles>

5a

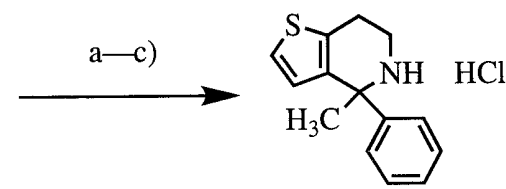

2a
Table 1. Yield of 2a by Each Method

\begin{tabular}{cc}
\hline \hline Method & Yield (\%) \\
\hline A & 2.5 \\
B & 17.3 \\
C & 15.2 \\
D & 27.9
\end{tabular}

a) $\mathrm{BF}_{3} \cdot \mathrm{Et}_{2} \mathrm{O} / \mathrm{THF}$ b) $\mathrm{CH}_{3} \mathrm{Li} / \mathrm{THF}$; c) $\mathrm{HCl} / \mathrm{EtOH}$

Chart 2

Method D<smiles>c1ccc(C2=NCCc3sccc32)cc1</smiles>

$5 a$

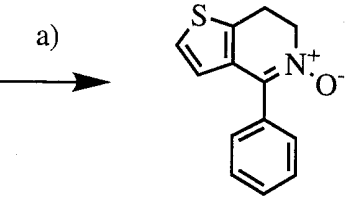

$8 a$ b)<smiles>CC1(c2ccccc2)c2ccsc2CCN1O</smiles>

$9 \mathbf{a}$<smiles>CC1(c2ccccc2)NCCc2sccc21</smiles>

2a

a) $m$-chloroperbenzoic acid $/ \mathrm{CH}_{2} \mathrm{Cl}_{2}$; b) $\mathrm{CH}_{3} \mathrm{MgBr} / \mathrm{THF}$; c) $\mathrm{PCl}_{3} / \mathrm{AcOEt}$; $) \mathrm{HCl} / \mathrm{EtOH}$

Chart 3

4,5,6,7-Tetrahydrothieno[3,2-c]pyridines $(\mathbf{2} \mathbf{a}, \mathbf{g}, \mathbf{i})$, 4,5,6,7-tetrahydrothieno[2,3-c]pyridine (2j) and 1,2,3,4tetrahydroisoquinolines (3a,e), in which the benzene ring in the isoquinoline nucleus and/or the $\mathrm{C}-1$ phenyl group of $( \pm)-1$ were replaced with thiophene rings, showed anticonvulsant activity at $100 \mathrm{mg} / \mathrm{kg}$ i.p., being comparably potent to $( \pm)-\mathbf{1}$. The result shows that the thiophene ring is a bioisostere of the benzene ring for anticonvulsant activity.

Optimal activity was associated with the C-1 methyl group ( $2 \mathbf{a}$ versus $\mathbf{2 b}, \mathbf{c}$ ), and the results were identical to the SAR for the isoquinoline derivatives of $( \pm)-\mathbf{1}^{1)}$ $N$-Alkylation (2d, e) was not tolerated. Replacing the C-1 phenyl group with a 2-furanyl group (3g) retained anticonvulsant activity, suggesting that a furan ring could also be a bioisostere for a benzene ring.
1,2,3,4-Tetrahydro[1] benzothieno[3,2-c]pyridine (4a) and 1,2,3,4-tetrahydropyrido[3,4-b]indoles $(\mathbf{4 b - \mathbf { b }})$ showed decreased anticonvulsant activity. The condensed rings may be too large to fit the NMDA receptor.

Since stereoisomers can exhibit different pharmacological properties, the optical isomers of $\mathbf{2} \mathbf{a}, \mathbf{g}$, and $\mathbf{3 a}$ were further evaluated for anticonvulsant activity against i.c.v. NMDA $(0.1 \mu \mathrm{g})$-induced seizures, anti-hypoxic effects and acute toxicity in mice. The results for these isomers, together with $(+)-\mathbf{1}($ FR115427), are presented in Table 3.

Optical isomers $(+)-\mathbf{2} \mathbf{a},(+)-\mathbf{2 g}$ and $(-)-\mathbf{3 a}$ showed anticonvulsant activity comparable to that of $(+)-1$ at $32 \mathrm{mg} / \mathrm{kg}$ i.p., but somewhat less potent than that of $(+)-1$ at a lower dose $(10 \mathrm{mg} / \mathrm{kg}$ i.p.). Compounds $(+)-\mathbf{2 a}, \mathbf{g}$ also showed significant anti-hypoxic activity at $3.2-10$ $\mathrm{mg} / \mathrm{kg}$ i.p. 
Table 2. Physical Properties and Anticonvulsant Activities for 4,5,6,7-Tetrahydrothieno[3,2-c]pyridines, 1,2,3,4-Tetrahydroisoquinolines, 1,2,3,4Tetrahydro[1]benzothieno[2,3-c]pyridine and Related Compounds<smiles>[R]N1CCc2ccsc2C1([R])[R]</smiles>

$2 a-k$

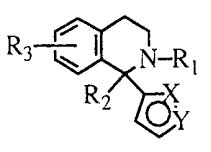

$3 \mathbf{a}-\mathbf{f}$

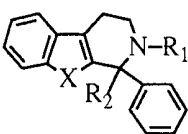

$\mathbf{4 a}-\mathbf{c}$

\begin{tabular}{|c|c|c|c|c|c|c|c|c|c|c|c|c|c|c|c|}
\hline \multirow{2}{*}{$\begin{array}{l}\text { Compd. } \\
\text { No. }\end{array}$} & \multirow[t]{2}{*}{$\mathrm{X}$} & \multirow[t]{2}{*}{$\mathrm{Y}$} & \multirow[t]{2}{*}{$\mathrm{R}_{1}$} & \multirow[t]{2}{*}{$\mathrm{R}_{2}$} & \multirow[t]{2}{*}{$\mathrm{R}_{3}$} & \multirow[t]{2}{*}{ Method } & \multirow{2}{*}{$\begin{array}{l}\text { Yield } \\
(\%)\end{array}$} & \multirow{2}{*}{$\begin{array}{c}\operatorname{mp}\left({ }^{\circ} \mathrm{C}\right) \\
\text { (Recryst. } \\
\text { solv.) }\end{array}$} & \multirow[t]{2}{*}{ Formula } & \multicolumn{3}{|c|}{$\begin{array}{l}\text { Analysis (\%) } \\
\text { Calcd (Found) }\end{array}$} & \multicolumn{3}{|c|}{$\begin{array}{c}\text { Anticonvulsant activity } \\
(\mathrm{s}) \\
(\mathrm{mg} / \mathrm{kg}, \text { i.p. })\end{array}$} \\
\hline & & & & & & & & & & C & $\mathrm{H}$ & $\mathrm{N}$ & 32 & 100 & 320 \\
\hline $2 a$ & $\mathrm{~S}$ & $\mathrm{CH}$ & $\mathrm{H}$ & $\mathrm{Ph}$ & $\mathrm{CH}_{3}$ & D & 50.4 & $\begin{array}{c}294 \\
(\mathrm{EtOH})\end{array}$ & $\mathrm{C}_{14} \mathrm{H}_{15} \mathrm{NS} \cdot \mathrm{HCl}$ & $\begin{array}{r}63.05 \\
(63.05\end{array}$ & $\begin{array}{l}6.07 \\
6.00\end{array}$ & $\begin{array}{l}5.27 \\
5.33)\end{array}$ & $14^{b)}$ & $>600^{c)}$ & \\
\hline $2 b$ & $\mathrm{~S}$ & $\mathrm{CH}$ & $\mathrm{H}$ & $\mathrm{Ph}$ & $\mathrm{H}$ & - & - & $\begin{array}{r}77-78^{d)} \\
(n \text {-hexane) }\end{array}$ & $\mathrm{C}_{13} \mathrm{H}_{13} \mathrm{NS}$ & & d) & & 9 & 13 & \\
\hline $2 c$ & $\mathrm{~S}$ & $\mathrm{CH}$ & $\mathrm{H}$ & $\mathrm{Ph}$ & $\mathrm{C}_{2} \mathrm{H}_{5}$ & B & 27.8 & $\begin{array}{c}285 \\
(\mathrm{EtOH})\end{array}$ & $\begin{array}{l}\mathrm{C}_{15} \mathrm{H}_{17} \mathrm{NS} \cdot \mathrm{HCl} \\
\cdot 0.1 \mathrm{H}_{2} \mathrm{O}\end{array}$ & $\begin{array}{r}63.97 \\
(63.97\end{array}$ & $\begin{array}{l}6.51 \\
6.57\end{array}$ & $\begin{array}{l}4.97 \\
4.92)\end{array}$ & 8 & $152^{e)}$ & $228^{b)}$ \\
\hline $2 d$ & $\mathrm{~S}$ & $\mathrm{CH}$ & $\mathrm{CH}_{3}$ & $\mathrm{Ph}$ & $\mathrm{CH}_{3}$ & A & 64.4 & $\begin{array}{c}239-240 \\
(\mathrm{EtOH})\end{array}$ & $\begin{array}{l}\mathrm{C}_{15} \mathrm{H}_{17} \mathrm{NS} \cdot \mathrm{HCl} \\
\cdot 0.25 \mathrm{H}_{2} \mathrm{O}\end{array}$ & $\begin{array}{r}63.34 \\
(63.72\end{array}$ & $\begin{array}{l}6.20 \\
6.45\end{array}$ & $\begin{array}{l}4.93 \\
4.91)\end{array}$ & 11 & $153^{e)}$ & $489^{e)}$ \\
\hline $2 e$ & $\mathrm{~S}$ & $\mathrm{CH}$ & $\mathrm{C}_{2} \mathrm{H}_{5}$ & $\mathrm{Ph}$ & $\mathrm{CH}_{3}$ & A & 67.9 & $\begin{array}{c}210-212 \\
\left(\mathrm{EtOH}-\mathrm{Et}_{2} \mathrm{O}\right)\end{array}$ & $\mathrm{C}_{16} \mathrm{H}_{19} \mathrm{NS} \cdot \mathrm{HCl}$ & $\begin{array}{r}65.40 \\
(65.21\end{array}$ & $\begin{array}{l}6.86 \\
7.03\end{array}$ & $\begin{array}{l}4.77 \\
4.76)\end{array}$ & 10 & $19^{e)}$ & $497^{e)}$ \\
\hline $2 f$ & $\mathrm{~S}$ & $\mathrm{CH}$ & $\mathrm{H}$ & $\mathrm{CH}_{2} \mathrm{Ph}$ & $\mathrm{CH}_{3}$ & C & 13.8 & $\begin{array}{c}206-208 \\
(\mathrm{EtOH})\end{array}$ & $\mathrm{C}_{15} \mathrm{H}_{17} \mathrm{NS} \cdot \mathrm{HCl}$ & $\begin{array}{r}64.38 \\
(64.04\end{array}$ & $\begin{array}{l}6.48 \\
6.54\end{array}$ & $\begin{array}{l}5.00 \\
4.96)\end{array}$ & $11^{b)}$ & $>600^{c)}$ & \\
\hline $2 \mathrm{~g}$ & $\mathrm{~S}$ & $\mathrm{CH}$ & $\mathrm{H}$ & 2-Thienyl & $\mathrm{CH}_{3}$ & D & 50.0 & 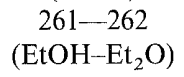 & $\mathrm{C}_{12} \mathrm{H}_{13} \mathrm{NS}_{2} \cdot \mathrm{HCl}$ & $\begin{array}{r}53.02 \\
(52.94\end{array}$ & $\begin{array}{l}5.19 \\
5.58\end{array}$ & $\begin{array}{l}5.15 \\
4.94)\end{array}$ & 15 & $>600^{c)}$ & $>600^{c)}$ \\
\hline $2 \mathrm{~h}$ & $\mathrm{~S}$ & $\mathrm{CH}$ & $\mathrm{CH}_{3}$ & 2-Thienyl & $\mathrm{CH}_{3}$ & A & 43.4 & 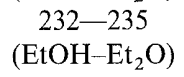 & $\begin{array}{l}\mathrm{C}_{13} \mathrm{H}_{15} \mathrm{NS}_{2} \cdot \mathrm{HCl} \\
\cdot 0.4 \mathrm{H}_{2} \mathrm{O}\end{array}$ & $\begin{array}{r}53.28 \\
(52.95\end{array}$ & $\begin{array}{l}5.78 \\
5.81\end{array}$ & $\begin{array}{l}4.78 \\
4.75)\end{array}$ & 11 & $19^{b)}$ & $>600^{c)}$ \\
\hline $2 \mathrm{i}$ & $\mathrm{S}$ & $\mathrm{CH}$ & $\mathrm{H}$ & 3-Thienyl & $\mathrm{CH}_{3}$ & $\mathrm{C}$ & 5.7 & $\begin{array}{c}235 \\
\left(\mathrm{EtOH}-\mathrm{Et}_{2} \mathrm{O}\right)\end{array}$ & $\mathrm{C}_{12} \mathrm{H}_{13} \mathrm{NS}_{2} \cdot \mathrm{HCl}$ & $\begin{array}{r}53.02 \\
(52.73\end{array}$ & $\begin{array}{l}5.19 \\
5.16\end{array}$ & $\begin{array}{l}5.15 \\
5.03)\end{array}$ & $21^{e)}$ & $>600^{c)}$ & \\
\hline $2 \mathbf{j}$ & $\mathrm{CH}$ & $\mathrm{S}$ & $\mathrm{H}$ & $\mathrm{Ph}$ & $\mathrm{CH}_{3}$ & D & 55.3 & 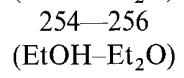 & $\begin{array}{l}\mathrm{C}_{14} \mathrm{H}_{15} \mathrm{NS} \cdot \mathrm{HCl} \\
\cdot 0.2 \mathrm{H}_{2} \mathrm{O}\end{array}$ & $\begin{array}{r}62.41 \\
(62.46\end{array}$ & $\begin{array}{l}6.13 \\
6.18\end{array}$ & $\begin{array}{l}5.19 \\
5.07)\end{array}$ & 9 & $>600^{\mathrm{c})}$ & \\
\hline $2 \mathrm{k}$ & $\mathrm{O}$ & $\mathrm{CH}$ & $\mathrm{H}$ & $\mathrm{Ph}$ & $\mathrm{CH}_{3}$ & A & 30.3 & 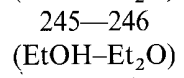 & $\begin{array}{l}\mathrm{C}_{14} \mathrm{H}_{15} \mathrm{NO} \cdot \mathrm{HCl} \\
\cdot 0.2 \mathrm{H}_{2} \mathrm{O}\end{array}$ & $\begin{array}{r}66.37 \\
(66.21\end{array}$ & $\begin{array}{l}6.52 \\
6.61\end{array}$ & $\begin{array}{l}5.52 \\
5.37)\end{array}$ & $14^{b)}$ & & \\
\hline $3 \mathbf{a}$ & S & $\mathrm{CH}$ & $\mathrm{H}$ & $\mathrm{CH}_{3}$ & $\mathrm{H}$ & B & 44.7 & 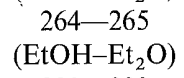 & $\begin{array}{l}\mathrm{C}_{14} \mathrm{H}_{15} \mathrm{NS} \cdot \mathrm{HCl} \\
\cdot 0.1 \mathrm{H}_{2} \mathrm{O}\end{array}$ & $\begin{array}{r}62.84 \\
(62.68\end{array}$ & $\begin{array}{l}6.10 \\
5.96\end{array}$ & $\begin{array}{l}5.23 \\
5.07)\end{array}$ & 25 & $482^{e)}$ & \\
\hline $3 \mathbf{b}$ & S & $\mathrm{CH}$ & $\mathrm{CH}_{3}$ & $\mathrm{CH}_{3}$ & $\mathrm{H}$ & A & 83.0 & $\begin{array}{c}220-222 \\
\left(\mathrm{EtOH}^{-}-\mathrm{Et}_{2} \mathrm{O}\right)\end{array}$ & $\begin{array}{l}\mathrm{C}_{15} \mathrm{H}_{17} \mathrm{NS} \cdot \mathrm{HCl} \\
\cdot 0.1 \mathrm{H}_{2} \mathrm{O}\end{array}$ & $\begin{array}{r}63.97 \\
(63.83\end{array}$ & $\begin{array}{l}6.51 \\
6.44\end{array}$ & $\begin{array}{l}4.97 \\
4.94)\end{array}$ & 13 & $168^{b)}$ & $>600^{c)}$ \\
\hline $3 c$ & $S$ & $\mathrm{CH}$ & $\mathrm{C}_{2} \mathrm{H}_{5}$ & $\mathrm{CH}_{3}$ & $\mathrm{H}$ & A & 66.2 & $\begin{array}{c}198-200 \\
\left(\mathrm{EtOH}-\mathrm{Et}_{2} \mathrm{O}\right)\end{array}$ & $\mathrm{C}_{16} \mathrm{H}_{19} \mathrm{NS} \cdot \mathrm{HCl}$ & $\begin{array}{r}65.40 \\
(65.29\end{array}$ & $\begin{array}{l}6.86 \\
6.78\end{array}$ & $\begin{array}{l}4.77 \\
5.07)\end{array}$ & 9 & $20^{b)}$ & 483 \\
\hline 3d & $\mathrm{S}$ & $\mathrm{CH}$ & $\mathrm{H}$ & $\mathrm{CH}_{3}$ & 6- $-\mathrm{CH}_{3} \mathrm{O}$ & B & 27.1 & $\begin{array}{c}286-287 \\
(\mathrm{EtOH})\end{array}$ & $\mathrm{C}_{15} \mathrm{H}_{17} \mathrm{NOS} \cdot \mathrm{HCl}$ & $\begin{array}{r}60.90 \\
(60.82\end{array}$ & $\begin{array}{l}6.13 \\
6.22\end{array}$ & $\begin{array}{l}4.73 \\
4.50)\end{array}$ & 21 & $>600^{c)}$ & \\
\hline $3 e$ & $\mathrm{CH}$ & $\mathrm{S}$ & $\mathrm{H}$ & $\mathrm{CH}_{3}$ & $\mathrm{H}$ & B & 42.1 & $\begin{array}{c}280-281 \\
(\mathrm{EtOH})\end{array}$ & $\mathrm{C}_{14} \mathrm{H}_{15} \mathrm{NS} \cdot \mathrm{HCl}$ & $\begin{array}{r}63.26 \\
(63.06\end{array}$ & $\begin{array}{l}6.06 \\
5.68\end{array}$ & $\begin{array}{l}5.26 \\
5.08)\end{array}$ & $147^{e)}$ & $>600^{c)}$ & \\
\hline $3 f$ & $\mathrm{O}$ & $\mathrm{CH}$ & $\mathrm{H}$ & $\mathrm{CH}_{3}$ & $\mathrm{H}$ & A & 53.9 & $\begin{array}{c}223-225 \\
(\mathrm{EtOH})\end{array}$ & $\mathrm{C}_{14} \mathrm{H}_{15} \mathrm{NO} \cdot \mathrm{HCl}$ & $\begin{array}{r}66.84 \\
(66.60\end{array}$ & $\begin{array}{l}6.49 \\
6.27\end{array}$ & $\begin{array}{l}5.56 \\
5.45)\end{array}$ & $26^{b)}$ & $>600^{c)}$ & \\
\hline $4 a$ & $\mathrm{~S}$ & - & H & $\mathrm{CH}_{3}$ & - & $\mathrm{D}$ & 54.6 & $\begin{array}{c}296-297 \\
\left(\mathrm{EtOH}-\mathrm{Et}_{2} \mathrm{O}\right)\end{array}$ & $\begin{array}{l}\mathrm{C}_{18} \mathrm{H}_{17} \mathrm{NS} \cdot \mathrm{HCl} \\
\cdot 0.5 \mathrm{H}_{2} \mathrm{O}\end{array}$ & $\begin{array}{r}66.54 \\
(66.78\end{array}$ & $\begin{array}{l}5.89 \\
5.73\end{array}$ & $\begin{array}{l}4.31 \\
4.20)\end{array}$ & 9 & 14 & $250^{b)}$ \\
\hline $4 b$ & $\mathrm{NH}$ & - & $\mathrm{H}$ & $\mathrm{CH}_{3}$ & - & A & 51.2 & $\begin{array}{c}267-269 \\
\left(\mathrm{EtOH}-\mathrm{Et}_{2} \mathrm{O}\right)\end{array}$ & $\begin{array}{l}\mathrm{C}_{18} \mathrm{H}_{18} \mathrm{~N}_{2} \cdot \mathrm{HCl} \\
0.6 \mathrm{H}_{2} \mathrm{O}\end{array}$ & $\begin{array}{r}69.83 \\
(69.47\end{array}$ & $\begin{array}{l}6.58 \\
6.48\end{array}$ & $\begin{array}{l}9.05 \\
9.35)\end{array}$ & $12^{b)}$ & 46 & \\
\hline $4 c$ & $\mathrm{NCH}_{3}$ & $3-$ & $\mathrm{H}$ & $\mathrm{CH}_{3}$ & - & A & 43.9 & 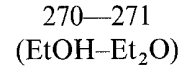 & $\begin{array}{l}\mathrm{C}_{19} \mathrm{H}_{20} \mathrm{~N}_{2} \cdot \mathrm{HCl} \\
0.1 \mathrm{H}_{2} \mathrm{O}\end{array}$ & $\begin{array}{r}72.53 \\
(72.20\end{array}$ & $\begin{array}{l}6.79 \\
6.86\end{array}$ & $\begin{array}{l}8.90 \\
8.63)\end{array}$ & 10 & 13 & $>600^{e)}$ \\
\hline$( \pm)-1$ & & & & & & & & & & & & & 52 & $>000$ & \\
\hline
\end{tabular}

a) Convulsions were induced by i.c.v NMDA $(0.32 \mu \mathrm{g})$. Each value represents the mean of 5 animals for the latency of initial seizure (s). $b$ ) $p<0.05$. c) $p<0.001 . \quad$ d) Lit., ${ }^{4)} \mathrm{mp} 88-89^{\circ} \mathrm{C}$. e) $p<0.01$. Values without superscripts are not statistically significantly different from the control.

The absolute configuration at the C-4 position of $(-)-3 \mathbf{a}$ was determined to be $R$ by a single-crystal X-ray analysis of $(-)$-3a $(+)$-di- $p$-toluoyl-D-tartarate (Fig. 2). This shows that $(-)-(R)-\mathbf{3 a}$ is the thiophene analog of $(+)-(S)-\mathbf{1}$, although the optical rotation of $(-)-3 \mathbf{a}$ was opposite to that of $(+)-(S)-1$.

In conclusion, 4,5,6,7-tetrahydrothieno[3,2-c]pyridines $((+)-\mathbf{2} \mathbf{a},(+)-\mathbf{2 g})$ and 1-(2-thienyl)-1,2,3,4-tetrahydroisoquinoline $((-)-3 \mathbf{a})$ exhibited cerebral protective activities such as in vivo NMDA antagonism and anti-hypoxic activity. The results also demonstrate that a thiophene ring is a bioisostere for the benzene ring with regard to anticonvulsant activity.

\section{Experimental}

Melting points were determined using a Thomas Hoover capillary melting point apparatus and are uncorrected. ${ }^{1} \mathrm{H}$-Nuclear magnetic resonance $\left({ }^{1} \mathrm{H}-\mathrm{NMR}\right)$ spectra were recorded at $90 \mathrm{MHz}$ on a Varian EM-390 NMR spectrometer using tetramethylsilane (TMS) as an internal standard. Infrared (IR) spectra were recorded on a Hitachi 260-10 spectrophotometer. Mass spectral (MS) measurements were made on a Hitachi M-80 or a JEOL-D300 mass spectrometer.

4-Phenyl-6,7-dihydrothieno[3,2-c]pyridine (5a) A mixture of $N$-[2-(2- 
Table 3. Physical Properties and Biological Data for Optical Isomers of $\mathbf{2 a}, \mathbf{g}$, and $\mathbf{3 a}$

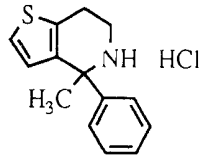

$\mathbf{2 a}$<smiles>CC1(c2cccs2)NCCc2sccc21</smiles>

$2 \mathrm{~g}$

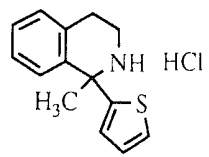

$3 \mathbf{a}$

\begin{tabular}{|c|c|c|c|c|c|c|c|c|c|c|c|c|}
\hline \multirow[t]{2}{*}{$\begin{array}{l}\text { Compound } \\
\text { No. }\end{array}$} & \multirow[t]{2}{*}{$\begin{array}{l}\text { Yield } \\
(\%)\end{array}$} & \multirow{2}{*}{$\begin{array}{c}\mathrm{mp}\left({ }^{\circ} \mathrm{C}\right) \\
\text { (Recryst. } \\
\text { solv.) }\end{array}$} & \multirow{2}{*}{$\begin{array}{c}{[\alpha]_{\mathrm{D}}^{25}} \\
(c=1 \\
\mathrm{MeOH}) \\
\text { (Degree) }\end{array}$} & \multirow[t]{2}{*}{ Formula } & \multicolumn{3}{|c|}{$\begin{array}{l}\text { Analysis (\%) } \\
\text { Caicd (Found) }\end{array}$} & \multicolumn{3}{|c|}{$\begin{array}{l}\text { Anticonvulsant } \\
\text { activity }{ }^{a)}(\mathrm{s}) \\
(\mathrm{mg} / \mathrm{kg}, \mathrm{i} . \mathrm{p} .)\end{array}$} & \multirow{2}{*}{$\begin{array}{l}\text { Anti-hypoxic } \\
\text { activity }^{b)} \\
\text { MED } \\
\text { (mg/kg, i.p.) }\end{array}$} & \multirow{2}{*}{$\begin{array}{c}\text { Acute } \\
\text { toxicity }^{c} \\
\mathrm{LD}_{50} \\
\text { (mg/kg, i.p.) }\end{array}$} \\
\hline & & & & & $\mathrm{C}$ & $\mathrm{H}$ & $\mathrm{N}$ & 10 & 32 & 100 & & \\
\hline$(+)-\mathbf{2 a}$ & 18.0 & $\begin{array}{c}295-297 \\
(\text { EtOH })\end{array}$ & +9.6 & $\begin{array}{l}\mathrm{C}_{14} \mathrm{H}_{15} \mathrm{NS} \cdot \mathrm{HCl} \\
\cdot 0.1 \mathrm{H}_{2} \mathrm{O}\end{array}$ & $\begin{array}{r}62.84 \\
62.86\end{array}$ & $\begin{array}{l}6.10 \\
6.12\end{array}$ & $\begin{array}{l}5.23 \\
5.26)\end{array}$ & $272^{d\rangle}$ & $>600^{e)}$ & & 10 & $>100<320$ \\
\hline$(-)-\mathbf{2 a}$ & 23.4 & $\begin{array}{c}278-280 \\
(\mathrm{EtOH})\end{array}$ & -4.6 & $\mathrm{C}_{14} \mathrm{H}_{15} \mathrm{NS} \cdot \mathrm{HCl}$ & $\begin{array}{r}63.26 \\
(62.95\end{array}$ & $\begin{array}{l}6.07 \\
5.98\end{array}$ & $\begin{array}{l}5.27 \\
5.09)\end{array}$ & 10 & 14 & $293^{d)}$ & NT & $>100<320$ \\
\hline$(+)-2 g$ & 29.5 & $\begin{array}{c}267-269 \\
(\mathrm{EtOH})\end{array}$ & +14.7 & $\begin{array}{l}\mathrm{C}_{12} \mathrm{H}_{13} \mathrm{NS}_{2} \cdot \mathrm{HCl} \\
\cdot 0.25 \mathrm{H}_{2} \mathrm{O}\end{array}$ & $\begin{array}{r}52.49 \\
(52.68\end{array}$ & $\begin{array}{l}5.42 \\
5.28\end{array}$ & $\begin{array}{l}4.98 \\
4.99)\end{array}$ & 25 & $>600^{d)}$ & $>600^{d)}$ & 3.2 & $>320$ \\
\hline$(-)-2 g$ & 21.1 & $\begin{array}{c}260-262 \\
(\mathrm{EtOH})\end{array}$ & -14.9 & $\begin{array}{l}\mathrm{C}_{12} \mathrm{H}_{13} \mathrm{NS}_{2} \cdot \mathrm{HCl} \\
\cdot 0.25 \mathrm{H}_{2} \mathrm{O}\end{array}$ & $\begin{array}{r}52.49 \\
(52.16\end{array}$ & $\begin{array}{l}5.42 \\
5.28\end{array}$ & $\begin{array}{l}4.98 \\
5.06)\end{array}$ & 28 & 28 & 37 & NT & $>320$ \\
\hline$(+)-\mathbf{3 a}$ & 13.3 & $\begin{array}{c}292-293 \\
(\mathrm{EtOH})\end{array}$ & +8.9 & $\mathrm{C}_{14} \mathrm{H}_{15} \mathrm{NS} \cdot \mathrm{HCl}$ & $\begin{array}{r}63.26 \\
(63.00\end{array}$ & $\begin{array}{l}6.07 \\
5.94\end{array}$ & $\begin{array}{l}5.27 \\
5.24)\end{array}$ & 8 & 14 & & NT & $>100<320$ \\
\hline$(-)-3 \mathbf{a}$ & 29.3 & $\begin{array}{c}289-290 \\
(\text { EtOH })\end{array}$ & -10.0 & $\begin{array}{l}\mathrm{C}_{14} \mathrm{H}_{15} \mathrm{NS} \cdot \mathrm{HCl} \\
\cdot 0.25 \mathrm{H}_{2} \mathrm{O}\end{array}$ & $\begin{array}{r}62.21 \\
(62.58\end{array}$ & $\begin{array}{l}6.15 \\
6.26\end{array}$ & $\begin{array}{l}5.18 \\
5.13)\end{array}$ & $159^{d\rangle}$ & $>600^{e)}$ & & NT & $>100<320$ \\
\hline $\begin{array}{c}(+)-1 \\
(\text { FR115427) }\end{array}$ & & & & & & & & $434^{d\rangle}$ & $>600^{d)}$ & & 3.2 & 200 \\
\hline
\end{tabular}

a) Convulsions were induced by i.c.v NMDA $(0.1 \mu \mathrm{g})$. Each value represents the mean of 5 animals for the latency of initial seizure (s). $b$ ) MED (minimum effective dose) is the dose showing a statistically significant effect ( $p<0.05$ using the Mann-Whitney $U$-test). $c$ ) Five ICR mice were used in each group. The median lethal dose $\left(\mathrm{LD}_{50}\right)$ value was calculated from the lethality within $7 \mathrm{~d}$ after an intraperitoneal administration of a test compound, using the Litchfield-Wilcoxon method. d) $p<0.01$. e) $p<0.001$. Values without superscripts are not statistically significantly different from the control. NT; not tested.

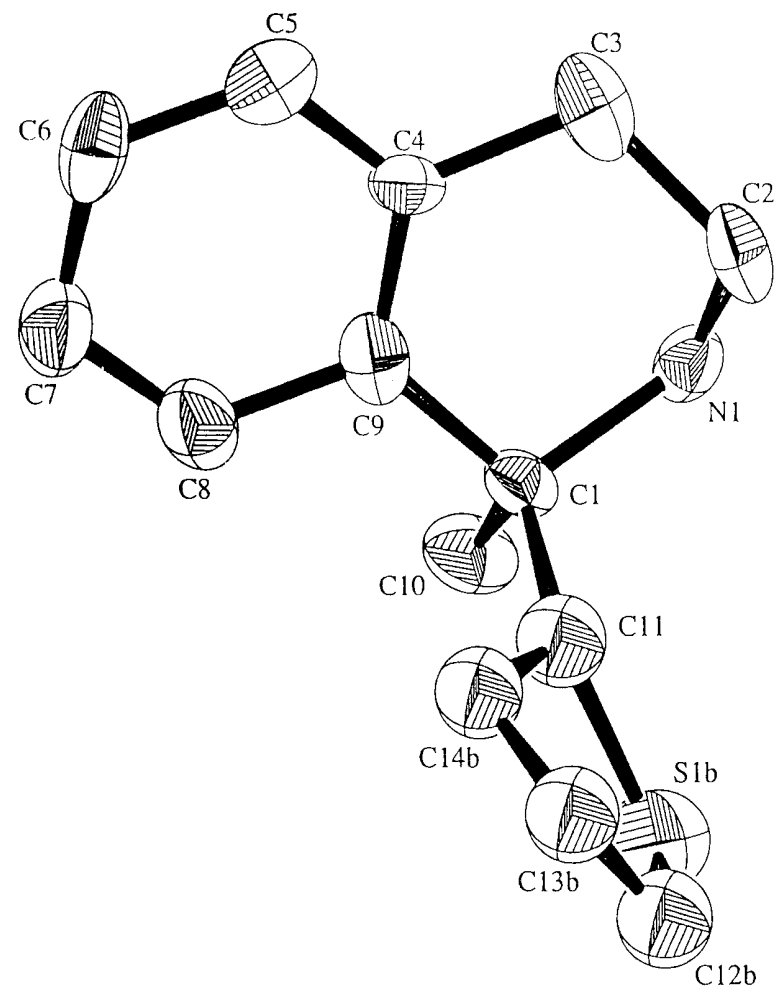

Fig. 2

thienyl)ethyl] benzamide ${ }^{3)}(2.0 \mathrm{~g}, 8.65 \mathrm{mmol}), \mathrm{P}_{2} \mathrm{O}_{5}(3.0 \mathrm{~g}, 21.1 \mathrm{mmol})$, and $\mathrm{POCl}_{3}(3.0 \mathrm{~g}, 19.6 \mathrm{mmol})$ in toluene $(20 \mathrm{ml})$ was refluxed for $3 \mathrm{~h}$. The solvent was decanted off, and the residual yellow oil was poured into water. The solution was adjusted to $\mathrm{pH} 10$ with saturated aqueous $\mathrm{K}_{2} \mathrm{CO}_{3}$, and extracted with ethyl acetate. The extract was washed with brine, dried over $\mathrm{MgSO}_{4}$, then evaporated in vacuo to give $5 \mathrm{a}(1.40 \mathrm{~g}$, $75.9 \%$ ) as a yellow oil [lit. ${ }^{3)}$ bp $149-152^{\circ} \mathrm{C}(0.5 \mathrm{~mm}$.)]. IR (film): 3050 , $1595,1570 \mathrm{~cm}^{-1} .{ }^{1} \mathrm{H}-\mathrm{NMR}\left(\mathrm{CDCl}_{3}\right) \delta: 2.93(2 \mathrm{H}, \mathrm{dd}, J=6.5,8.3 \mathrm{~Hz})$,
$3.95(2 \mathrm{H}, \mathrm{dd}, J=6.5,8.3 \mathrm{~Hz}), 7.01(1 \mathrm{H}, \mathrm{d}, J=5.2 \mathrm{~Hz}), 7.08(1 \mathrm{H}, \mathrm{d}$, $J=5.2 \mathrm{~Hz}), 7.26-7.48(3 \mathrm{H}, \mathrm{m}), 7.62-7.71(2 \mathrm{H}, \mathrm{m}) . \mathrm{MS} m / z: 213\left(\mathrm{M}^{+}\right)$. The following compounds were prepared similarly, and were not further purified or analyzed before use in the next step.

4-Benzyl-6,7-dihydrothieno[3,2-c]pyridine (5b): $\mathbf{5 b}$ was prepared from $N$-[2-(2-thienyl)ethyl]phenylethanamide ${ }^{5)}$ in $77.7 \%$ yield as a yellow oil. IR (film): $1620,1600 \mathrm{~cm}^{-1} .{ }^{1} \mathrm{H}-\mathrm{NMR}\left(\mathrm{CDCl}_{3}\right) \delta: 2.81-2.89(2 \mathrm{H}, \mathrm{m})$, $3.86-3.90(2 \mathrm{H}, \mathrm{m}), 3.96(2 \mathrm{H}, \mathrm{s}), 7.09-7.35(7 \mathrm{H}, \mathrm{m})$.

4-(2-Thienyl)-6,7-dihydrothieno[3,2-c]pyridine (5c): $\mathbf{5 c}$ was prepared from $N$-[2-(2-thienyl)ethyl]-2-thiophenecarboxamide (mp 97-98 ${ }^{\circ} \mathrm{C}$ ), which was obtained by acylation of 2-(2-thienyl)ethylamine with 2 -thiophenecarbonyl chloride, in $85.8 \%$ yield as a yellow oil. IR (film): $1585,1510 \mathrm{~cm}^{-1} .{ }^{1} \mathrm{H}-\mathrm{NMR}\left(\mathrm{CDCl}_{3}\right) \delta: 2.85-2.93(2 \mathrm{H}, \mathrm{m}), 3.85-3.93$ $(2 \mathrm{H}, \mathrm{m}), 7.08-7.16(2 \mathrm{H}, \mathrm{m}), 7.33(1 \mathrm{H}, \mathrm{d}, J=5.2 \mathrm{~Hz}), 7.41(1 \mathrm{H}, \mathrm{d}$, $J=5.2 \mathrm{~Hz}), 7.47-7.50(1 \mathrm{H}, \mathrm{m})$.

4-(3-Thienyl)-6,7-dihydrothieno[3,2-c]pyridine (5d): $5 \mathbf{d}$ was prepared from $N$-[2-(2-thienyl)ethyl]-3-thiophenecarboxamide, which was obtained by acylation of 2-(2-thienyl)ethylamine with 3-thiophenecarbonyl chloride and was not isolated before use in the next step, in $90.4 \%$ yield as a yellow oil. IR (film): $1580,1510 \mathrm{~cm}^{-1}$. ${ }^{1} \mathrm{H}-\mathrm{NMR}\left(\mathrm{CDCl}_{3}\right) \delta$ : $2.86-2.93(2 \mathrm{H}, \mathrm{m}), 3.86-3.94(2 \mathrm{H}, \mathrm{m}), 7.11(1 \mathrm{H}, \mathrm{d}, J=5.2 \mathrm{~Hz}), 7.19$ $(1 \mathrm{H}, \mathrm{d}, J=5.2 \mathrm{~Hz}), 7.36(1 \mathrm{H}, \mathrm{d}, J=4.6 \mathrm{~Hz}), 7.51(1 \mathrm{H}, \mathrm{d}, J=4.6 \mathrm{~Hz}), 7.66$ $(1 \mathrm{H}, \mathrm{s})$.

4-Phenyl-6,7-dihydrothieno[2,3-c]pyridine $(\mathbf{5 e})^{3)}: \mathbf{5 e}$ was prepared from $N$-[2-(3-thienyl)ethyl]benzamide ${ }^{3)}$ in $65.0 \%$ yield as a yellow oil [lit. ${ }^{2)}$ bp $\left.150{ }^{\circ} \mathrm{C}(0.5 \mathrm{~mm})\right] .{ }^{1} \mathrm{H}-\mathrm{NMR}\left(\mathrm{CDCl}_{3}\right) \delta: 2.82(2 \mathrm{H}, \mathrm{dd}, J=6.5,8.4 \mathrm{~Hz})$, $3.93(2 \mathrm{H}, \mathrm{dd}, J=6.5,8.4 \mathrm{~Hz}), 7.00(1 \mathrm{H}, \mathrm{d}, J=4.9 \mathrm{~Hz}), 7.25-7.49(4 \mathrm{H}$, m), $7.76-7.85(2 \mathrm{H}, \mathrm{m}) . \mathrm{MS} m / z: 213\left(\mathrm{M}^{+}\right)$.

4-Phenyl-6,7-dihydrofuro[3,2-c]pyridine $(\mathbf{5 f})$ : $\mathbf{5 f}$ was prepared from $N$-[2-(2-furyl)ethyl]benzamide $\left(\mathrm{mp} 69-70^{\circ} \mathrm{C}\right)$, which was obtained by acylation of 2-(2-furyl)ethylamine ${ }^{6)}$ with benzoyl chloride, in $35.4 \%$ yield as a yellow oil. IR (film): $1600 \mathrm{~cm}^{-1} .{ }^{1} \mathrm{H}-\mathrm{NMR}\left(\mathrm{CDCl}_{3}\right) \delta: 2.81-2.87$ $(2 \mathrm{H}, \mathrm{m}), 4.02-4.11(2 \mathrm{H}, \mathrm{m}), 6.50(1 \mathrm{H}, \mathrm{d}, J=2 \mathrm{~Hz}), 7.35(1 \mathrm{H}, \mathrm{d}, J=2 \mathrm{~Hz})$, $7.40-7.47(3 \mathrm{H}, \mathrm{m}), 7.71-7.94(2 \mathrm{H}, \mathrm{m})$. MS $m / z: 197\left(\mathrm{M}^{+}\right)$.

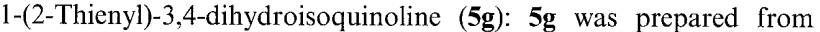
$N$-phenethyl-2-thiophenecarboxamide ${ }^{7)}$ in $90.0 \%$ yield as a yellow oil. IR (film): $1600,1560 \mathrm{~cm}^{-1}$.

1-(3-Thienyl)-3,4-dihydroisoquinoline (5h): $5 \mathbf{h}$ was prepared from $N$-phenethyl-3-thiophenecarboxamide (mp $104-105^{\circ} \mathrm{C}$ ), which was obtained by acylation of phenethylamine with 3-thiophenecarbonyl 
chloride, in $76.2 \%$ yield as a yellow oil. IR (film): $3100,3060,1600$ $1560 \mathrm{~cm}^{-1}$

1-(2-Furanyl)-3,4-dihydroisoquinoline (5i): $\mathbf{5 i}$ was prepared from $\mathrm{N}$-phenethyl-2-furancarboxamide $\left(\mathrm{mp} 43-45^{\circ} \mathrm{C}\right.$ ), which was obtained by acylation of phenethylamine with 2-furancarbonyl chloride, in $84.3 \%$ yield as a yellow oil. IR (film): $3500,1600,1560 \mathrm{~cm}^{-1}$.

1-Phenyl-3,4-dihydro[1]benzothieno[2,3-c]pyridine $(\mathbf{5 j})^{8)}$ : $\mathbf{5 j}$ was prepared from $N$-[2-(3-benzo[b]thienyl)ethyl] benzamide ${ }^{8)}$ in $83.9 \%$ yield as a pale yellow solid, mp $59-60^{\circ} \mathrm{C}\left(n\right.$-pentane). ${ }^{1} \mathrm{H}-\mathrm{NMR}\left(\mathrm{CDCl}_{3}\right)$ $\delta: 2.87(2 \mathrm{H}, \mathrm{dd}, J=7,8 \mathrm{~Hz}), 3.98(2 \mathrm{H}, \mathrm{dd}, J=7,8 \mathrm{~Hz}), 7.23-7.38(5 \mathrm{H}$, $\mathrm{m}), 7.65-7.80(4 \mathrm{H}, \mathrm{m})$. MS $m / z: 263\left(\mathrm{M}^{+}\right)$.

1-(2-Thienyl)-3,4-dihydro-6-methoxyisoquinoline (5k) was not isolated before use in the next step.

9H-1-phenyl-3,4-dihydropyrido[3,4-b]indole (51): 51 was prepared from $N$-[2-(3-indolyl)ethyl]benzamide ${ }^{9}$ in $64.6 \%$ yield as a pale yellow solid, $\mathrm{mp} 205-207^{\circ} \mathrm{C}$ (ether). IR (Nujol): $1620,1590,1560,1535 \mathrm{~cm}^{-1}$ ${ }^{1} \mathrm{H}-\mathrm{NMR}$ (DMSO- $d_{6}$ ) $\delta: 2.82-2.91(2 \mathrm{H}, \mathrm{m}), 3.85-3.93(2 \mathrm{H}, \mathrm{m})$, $7.04-7.24(2 \mathrm{H}, \mathrm{m}), 7.43(1 \mathrm{H}, \mathrm{d}, J=8.1 \mathrm{~Hz}), 7.49-7.54(3 \mathrm{H}, \mathrm{m}), 7.61$ $(1 \mathrm{H}, \mathrm{d}, J=8.1 \mathrm{~Hz}), 7.74-7.79(2 \mathrm{H}, \mathrm{m})$.

Methods A-C. 5-Benzyl-4-phenyl-6,7-dihydrothieno[3,2-c]pyridinium Iodide (6a) Benzyl iodide $(1.72 \mathrm{~g}, 7.88 \mathrm{mmol})$ was added to a solution of 4-phenyl-6,7-dihydrothieno[3,2-c]pyridine $(5 \mathrm{a})^{3)}(1.4 \mathrm{~g}, 6.56 \mathrm{mmol})$ in $\mathrm{CH}_{3} \mathrm{CN}(14 \mathrm{ml})$, and the mixture was refluxed for $1 \mathrm{~h}$. It was allowed to cool to room temperature, and the solvent was evaporated in vacuo. The residue was washed with ether and ethyl acetate, and then recrystallized from a solution of $\mathrm{CH}_{3} \mathrm{CN}$ and ether to give $6 \mathrm{a}(63.6 \%, 1.80 \mathrm{~g})$ as a yellow solid, mp $165-167^{\circ} \mathrm{C}$. IR (Nujol): $1605,1310 \mathrm{~cm}^{-1} \cdot{ }^{1} \mathrm{H}-\mathrm{NMR}$ $\left(\right.$ DMSO- $\left.d_{6}\right) \delta: 3.58(2 \mathrm{H}, \mathrm{d}, J=8 \mathrm{~Hz}), 4.32(2 \mathrm{H}, \mathrm{d}, J=8 \mathrm{~Hz}), 5.16(2 \mathrm{H}$ s), $6.78(1 \mathrm{H}, \mathrm{d}, J=6 \mathrm{~Hz}), 7.50(5 \mathrm{H}, \mathrm{s}), 7.73(1 \mathrm{H}, \mathrm{d}, J=6 \mathrm{~Hz}), 7.84(5 \mathrm{H}$, s). The following compounds were prepared similarly, and were not further purified or analyzed before use in the next step.

5-(4-Methoxyphenylmethyl)-4-phenyl-6,7-dihydrothieno[3,2-c]pyridinium Iodide $(\mathbf{6 b})$ : $\mathbf{6 b}$ was obtained from $5 \mathbf{a}(5.0 \mathrm{~g}, 23.4 \mathrm{mmol})$ and 4-methoxyphenylmethyl iodide ( $6.98 \mathrm{~g}, 28.1 \mathrm{mmol})$ in $100 \%$ yield $(10.8 \mathrm{~g})$ as a yellow oil. IR (film): $1600,1500 \mathrm{~cm}^{-1} \cdot{ }^{1} \mathrm{H}-\mathrm{NMR}$ (DMSO- $\left.d_{6}\right) \delta$ $3.32(3 \mathrm{H}, \mathrm{s}), 3.42-3.65(2 \mathrm{H}, \mathrm{m}), 3.80(2 \mathrm{H}, \mathrm{s}), 4.20-4.48(2 \mathrm{H}, \mathrm{m})$, $6.76-7.82(11 \mathrm{H}, \mathrm{m})$.

5-Methyl-4-phenyl-6,7-dihydrothieno[3,2-c]pyridinium Iodide (6c): $\mathbf{6 c}$ was obtained from $5 \mathrm{a}(4.0 \mathrm{~g}, 18.8 \mathrm{mmol})$ and methyl iodide $(4.67 \mathrm{ml}$, $75.0 \mathrm{mmol})$ in $68.9 \%$ yield $(4.60 \mathrm{~g})$ as a yellow solid, $169-176^{\circ} \mathrm{C}$ (acetone). IR (Nujol): $1630,1600,1510 \mathrm{~cm}^{-1} .{ }^{1} \mathrm{H}-\mathrm{NMR}$ (DMSO- $d_{6}$ ) $\delta$ : $3.34-3.75(2 \mathrm{H}, \mathrm{m}), 3.50(3 \mathrm{H}, \mathrm{s}), 4.26-5.53(2 \mathrm{H}, \mathrm{m}), 6.70(1 \mathrm{H}, \mathrm{d}$, $J=5 \mathrm{~Hz}), 7.62(1 \mathrm{H}, \mathrm{d}, J=5 \mathrm{~Hz}), 7.68-7.77(5 \mathrm{H}, \mathrm{m})$.

5-Ethyl-4-phenyl-6,7-dihydrothieno[3,2-c]pyridinium Iodide (6d): $6 \mathbf{d}$ was obtained from $\mathbf{5 a}(3.0 \mathrm{~g}, 14.1 \mathrm{mmol})$ and ethyl iodide $(5.6 \mathrm{ml}$, $70.3 \mathrm{mmol})$ in $90.1 \%$ yield $(4.68 \mathrm{~g})$ as a yellow solid, $134-136^{\circ} \mathrm{C}$ (acetone). IR (Nujol): $1595,1500 \mathrm{~cm}^{-1} .{ }^{1} \mathrm{H}-\mathrm{NMR}$ (DMSO- $d_{6}$ ) $\delta: 1.37$ $(3 \mathrm{H}, \mathrm{t}, J=7 \mathrm{~Hz}), 3.34-4.00(4 \mathrm{H}, \mathrm{m}), 4.42(2 \mathrm{H}, \mathrm{q}, J=7 \mathrm{~Hz}), 6.70(1 \mathrm{H}$, $\mathrm{d}, J=5 \mathrm{~Hz}), 7.69(1 \mathrm{H}, \mathrm{d}, J=5 \mathrm{~Hz}), 7.76-7.86(5 \mathrm{H}, \mathrm{m})$.

5-Methyl-4-(2-thienyl)-6,7-dihydrothieno[3,2-c]pyridinium Iodide (6e): 6e was obtained from $5 \mathrm{c}(3.6 \mathrm{~g}, 16.4 \mathrm{mmol})$ and methyl iodide $(4.09 \mathrm{ml}, 65.7 \mathrm{mmol})$ in $80.3 \%$ yield $(4.76 \mathrm{~g})$ as a yellow solid, $165-168^{\circ} \mathrm{C}$ (acetone). IR (Nujol): $1580 \mathrm{~cm}^{-1} .{ }^{1} \mathrm{H}-\mathrm{NMR}$ (DMSO- $d_{6}$ ) $\delta: 3.30-3.60$ $(2 \mathrm{H}, \mathrm{m}), 3.67(3 \mathrm{H}, \mathrm{s}), 4.18-4.36(2 \mathrm{H}, \mathrm{m}), 6.92(1 \mathrm{H}, \mathrm{d}, J=5 \mathrm{~Hz})$, $7.38-8.30(4 \mathrm{H}, \mathrm{m})$.

2-(4-Methoxyphenylmethyl)-1-(2-thienyl)-3,4-dihydroisoquinolinium Iodide (6f): 6f was obtained from $5 \mathrm{~g}(5.0 \mathrm{~g}, 23.4 \mathrm{mmol})$ and 4 -methoxyphenylmethyl iodide $(6.98 \mathrm{~g}, 28.1 \mathrm{mmol})$ in $100 \%$ yield $(10.8 \mathrm{~g})$ as a yellow oil. IR (film): $1610,1570,1500 \mathrm{~cm}^{-1} .{ }^{1} \mathrm{H}-\mathrm{NMR}$ (DMSO- $d_{6}$ ) $\delta: 2.98-3.22(2 \mathrm{H}, \mathrm{m}), 3.75(3 \mathrm{H}, \mathrm{s}), 3.75-4.04(2 \mathrm{H}, \mathrm{m}), 5.17(2 \mathrm{H}, \mathrm{s})$, $6.80(1 \mathrm{H}, \mathrm{d}, J=6 \mathrm{~Hz}), 7.04-8.23(10 \mathrm{H}, \mathrm{m})$.

2-Methyl-1-(2-thienyl)-3,4-dihydroisoquinolinium Iodide (6g): $\mathbf{6 g}$ was obtained from $5 \mathrm{~g}(3.0 \mathrm{~g}, 14.1 \mathrm{mmol})$ and methyl iodide $(3.5 \mathrm{ml}, 56.3 \mathrm{mmol})$ in $39.2 \%$ yield $\left(1.96 \mathrm{~g}\right.$ ) as a yellow solid, $\mathrm{mp} 180-181^{\circ} \mathrm{C}$ (acetone). IR (Nujol): $1590 \mathrm{~cm}^{-1} .{ }^{1} \mathrm{H}-\mathrm{NMR}$ (DMSO- $d_{6}$ ) $\delta: 3.25-3.54(2 \mathrm{H}, \mathrm{m}), 3.75$ $(3 \mathrm{H}, \mathrm{s}), 4.22-4.48(2 \mathrm{H}, \mathrm{m}), 7.24-7.88(6 \mathrm{H}, \mathrm{m}), 8.37(1 \mathrm{H}, \mathrm{d}, J=7 \mathrm{~Hz})$.

2-Ethyl-1-(2-thienyl)-3,4-dihydroisoquinolinium Iodide (6h): $6 \mathbf{h}$ was obtained from $5 \mathrm{~g}(3.0 \mathrm{~g}, 14.1 \mathrm{mmol})$ and ethyl iodide $(5.6 \mathrm{ml}, 70.3 \mathrm{mmol})$ in $54.5 \%$ yield $(1.96 \mathrm{~g})$ as a yellow solid, $\mathrm{mp} 194-195^{\circ} \mathrm{C}$ (acetone). IR (Nujol): $1600,1560,1520 \mathrm{~cm}^{-1} .{ }^{1} \mathrm{H}-\mathrm{NMR}$ (DMSO- $\left.d_{6}\right) \delta: 1.43(3 \mathrm{H}, \mathrm{t}$, $J=8 \mathrm{~Hz}), 3.25-3.48(2 \mathrm{H}, \mathrm{m}), 3.76-4.08(2 \mathrm{H}, \mathrm{m}), 4.25(2 \mathrm{H}, \mathrm{q}, J=8 \mathrm{~Hz})$, $7.12-7.83(6 \mathrm{H}, \mathrm{m}), 8.24(1 \mathrm{H}, \mathrm{d}, J=7 \mathrm{~Hz})$.

5-Benzyl-4-phenyl-6,7-dihydrofuro[3,2-c]pyridinium iodide (6i), 2-(4methoxyphenylmethyl)-1-(2-thienyl)-3,4-dihydroisoquinolinium iodide (6j), 2-(4-methoxyphenylmethyl)-1-(3-thienyl)-3,4-dihydroisoquinolinium iodide (6k), 2-(4-methoxyphenylmethyl)-1-(2-furanyl)-3,4-dihydroisoquinolinium iodide (61), and 2-benzyl-3,4-dihydro-1-phenyl-pyrido $[3,4-b]$ indolinium iodide $(\mathbf{6 m})$ were not isolated before use in the next step.

5-Benzyl-4-methyl-4-phenyl-6,7-dihydrothieno[3,2-c]pyridine (7a) A solution of $3 \mathrm{M}$ methylmagnesium bromide in ether $(4.6 \mathrm{ml}, 13.9 \mathrm{mmol})$ was added to a suspension of $6 \mathbf{a}(1.5 \mathrm{~g}, 3.48 \mathrm{mmol})$ in ether $(20 \mathrm{ml})$ at room temperature, and the whole was refluxed for $1 \mathrm{~h}$. It was allowed to cool to room temperature, poured into saturated aqueous $\mathrm{NH}_{4} \mathrm{Cl}$, then extracted with ethyl acetate. The extract was washed with water, brine, dried over $\mathrm{MgSO}_{4}$, and evaporated in vacuo to give $7 \mathbf{a}$ in $64.8 \%$ yield $(0.72 \mathrm{~g}, 2.25 \mathrm{mmol})$ as a colorless oil. IR (film): $1600,1490 \mathrm{~cm}^{-1}$. ${ }^{1} \mathrm{H}-\mathrm{NMR}\left(\mathrm{CDCl}_{3}\right) \delta: 1.80(3 \mathrm{H}, \mathrm{s}), 2.82-2.93(4 \mathrm{H}, \mathrm{m}), 3.43(1 \mathrm{H}, \mathrm{d}$, $J=14 \mathrm{~Hz}), 3.57(1 \mathrm{H}, \mathrm{d}, J=14 \mathrm{~Hz}), 6.37(1 \mathrm{H}, \mathrm{d}, J=5 \mathrm{~Hz}), 6.93(1 \mathrm{H}, \mathrm{d}$, $J=5 \mathrm{~Hz}), 7.28-7.85(10 \mathrm{H}, \mathrm{m})$.

Compounds $\mathbf{2 d}, \mathbf{e}, \mathbf{h}$, and $\mathbf{3 b}, \mathbf{c}$ were similarly prepared from $\mathbf{6 c -} \mathbf{e}$, $\mathbf{g}, \mathbf{h}$, respectively. Physical properties and spectral data for these compounds are listed in Tables 2 and 4 . The following compounds were also prepared by the same procedures as employed for the preparation of 7a and were not further purified or analyzed before use in the next step.

5-(4-Methoxyphenylmethyl)-4-methyl-4-phenyl-4,5,6,7-tetrahydrothieno[3,2-c]pyridine $(7 \mathbf{b}): 7 \mathbf{b}$ was obtained from $\mathbf{6 b}(12.0 \mathrm{~g}, 26.0 \mathrm{mmol})$ in $87.7 \%$ yield $(7.97 \mathrm{~g})$ as a pale yellow oil. IR (film): 1600,1580 , $1500 \mathrm{~cm}^{-1} .{ }^{1} \mathrm{H}-\mathrm{NMR}\left(\mathrm{CDCl}_{3}\right) \delta: 1.77(3 \mathrm{H}, \mathrm{s}), 2.82-2.93(4 \mathrm{H}, \mathrm{m}), 3.30$ $(1 \mathrm{H}, \mathrm{d}, J=14 \mathrm{~Hz}), 3.50(1 \mathrm{H}, \mathrm{d}, J=14 \mathrm{~Hz}), 3.78(3 \mathrm{H}, \mathrm{s}), 6.38(1 \mathrm{H}, \mathrm{d}$, $J=7 \mathrm{~Hz}), 6.76-7.70(10 \mathrm{H}, \mathrm{m})$.

5-Benzyl-4-methyl-4-phenyl-4,5,6,7-tetrahydrofuro[3,2-c]pyridine (7c): $7 \mathbf{c}$ was obtained from $\mathbf{6 i}$ in $21.7 \%$ yield $(0.1 \mathrm{~g})$ as a white solid, $\mathrm{mp}$ $90-91{ }^{\circ} \mathrm{C}$ ( $n$-hexane). IR (Nujol): $1500 \mathrm{~cm}^{-1} .{ }^{1} \mathrm{H}-\mathrm{NMR}\left(\mathrm{CDCl}_{3}\right) \delta: 1.72$ $(3 \mathrm{H}, \mathrm{s}), 2.54-2.88(4 \mathrm{H}, \mathrm{m}), 3.38(1 \mathrm{H}, \mathrm{d}, J=13.8 \mathrm{~Hz}), 3.52(1 \mathrm{H}, \mathrm{d}$, $J=13.8 \mathrm{~Hz}), 5.87(1 \mathrm{H}, \mathrm{d}, J=2.0 \mathrm{~Hz}), 7.17-7.36(9 \mathrm{H}, \mathrm{m}), 7.57(1 \mathrm{H}, \mathrm{d}$, $J=7.6 \mathrm{~Hz}) . \mathrm{MS} m / z: 303\left(\mathrm{M}^{+}\right)$.

2-(4-Methoxyphenylmethyl)-1-methyl-1-(2-thienyl)-1,2,3,4-tetrahydroisoquinoline (7d): $7 \mathbf{d}$ was obtained from 6 f in $47.1 \%$ yield $(4.28 \mathrm{~g})$ as a white solid, $\mathrm{mp} 80-82^{\circ} \mathrm{C}\left(\mathrm{EtOH}-\mathrm{Et}_{2} \mathrm{O}\right)$. IR (Nujol): 1610,1580 , $1510 \mathrm{~cm}^{-1} .{ }^{1} \mathrm{H}-\mathrm{NMR}\left(\mathrm{CDCl}_{3}\right) \delta: 1.81(3 \mathrm{H}, \mathrm{s}), 2.48-3.05(4 \mathrm{H}, \mathrm{m}), 3.30$ $(1 \mathrm{H}, \mathrm{d}, J=14 \mathrm{~Hz}), 3.68(1 \mathrm{H}, \mathrm{d}, J=14 \mathrm{~Hz}), 3.72(3 \mathrm{H}, \mathrm{s}), 6.70-7.31(11 \mathrm{H}$, $\mathrm{m})$.

2-(4-Methoxyphenylmethyl)-1-methyl-6-methoxy-1-(2-thienyl)1,2,3,4-tetrahydroisoquinoline (7e): $7 \mathbf{e}$ was obtained from $6 \mathbf{j}$ in $60.9 \%$ yield $(5.25 \mathrm{~g})$ as a pale yellow oil. IR (film): $1605,1580,1500 \mathrm{~cm}^{-1}$. ${ }^{1} \mathrm{H}-\mathrm{NMR}\left(\mathrm{CDCl}_{3}\right) \delta: 1.81(3 \mathrm{H}, \mathrm{s}), 2.59-3.02(4 \mathrm{H}, \mathrm{m}), 3.36(1 \mathrm{H}, \mathrm{d}$, $J=13.6 \mathrm{~Hz}), 3.70(1 \mathrm{H}, \mathrm{d}, J=14 \mathrm{~Hz}), 3.74(3 \mathrm{H}, \mathrm{s}), 3.79(3 \mathrm{H}, \mathrm{s}), 6.57-6.64$ $(2 \mathrm{H}, \mathrm{m}), 6.81-6.92(4 \mathrm{H}, \mathrm{m}), 7.05-7.36(4 \mathrm{H}, \mathrm{m})$.

2-(4-Methoxyphenylmethyl)-1-methyl-1-(3-thienyl)-1,2,3,4-tetrahydroisoquinoline (7f): $7 f$ was obtained from $6 \mathbf{k}$ in $64.4 \%$ yield $(5.27 \mathrm{~g})$ as a pale yellow oil. IR (film): $1610,1580,1500 \mathrm{~cm}^{-1} .{ }^{1} \mathrm{H}-\mathrm{NMR}\left(\mathrm{CDCl}_{3}\right)$ $\delta: 1.78(3 \mathrm{H}, \mathrm{s}), 2.5 \mathrm{l}-3.05(4 \mathrm{H}, \mathrm{m}), 3.25(1 \mathrm{H}, \mathrm{d}, J=13.5 \mathrm{~Hz}), 3.45(1 \mathrm{H}$, d, $J=13.5 \mathrm{~Hz}), 3.78(3 \mathrm{H}, \mathrm{s}), 6.61-6.65(2 \mathrm{H}, \mathrm{m}), 6.75-7.29(9 \mathrm{H}, \mathrm{m})$.

2-Benzyl-1-methyl-1-(2-furanyl)-1,2,3,4-tetrahydroisoquinoline (7g): $7 \mathrm{~g}$ was obtained from $6 \mathrm{l}$ in $59.4 \%$ yield $(0.9 \mathrm{~g})$ as a pale yellow oil. IR (film): $1600,1580,1500 \mathrm{~cm}^{-1} \cdot{ }^{1} \mathrm{H}-\mathrm{NMR}\left(\mathrm{CDCl}_{3}\right) \delta: 1.83(3 \mathrm{H}, \mathrm{s})$, $2.84-3.10(4 \mathrm{H}, \mathrm{m}), 3.46(1 \mathrm{H}, \mathrm{d}, J=14 \mathrm{~Hz}), 3.82(1 \mathrm{H}, \mathrm{d}, J=14 \mathrm{~Hz})$, $6.32-6.42(2 \mathrm{H}, \mathrm{m}), 7.05-7.50(10 \mathrm{H}, \mathrm{m})$.

2-Benzyl-1-methyl-1-phenyl-1,2,3,4-tetrahydropyridino[3,4-b]indole (7h): $7 \mathrm{~h}$ was obtained from $6 \mathrm{~m}$ in $99.0 \%$ yield $(0.75 \mathrm{~g})$ as a pale yellow oil. IR (film): $3400,1600 \mathrm{~cm}^{-1}$. ${ }^{1} \mathrm{H}-\mathrm{NMR}\left(\mathrm{CDCl}_{3}\right) \delta: 1.85(3 \mathrm{H}, \mathrm{s})$, $2.75-2.93(4 \mathrm{H}, \mathrm{m}), 3.38(1 \mathrm{H}, \mathrm{d}, J=14 \mathrm{~Hz}), 3.63(1 \mathrm{H}, \mathrm{d}, J=14 \mathrm{~Hz})$, $6.98-7.62(14 \mathrm{H}, \mathrm{m})$. MS $m / z: 352\left(\mathrm{M}^{+}\right)$.

2-Benzyl-1,9-dimethyl-1-phenyl-1,2,3,4-tetrahydropyridino $[3,4-b]$ indole $(7 \mathrm{i}) \mathrm{Na}(0.09 \mathrm{~g}, 3.97 \mathrm{mmol})$ was dissolved in liquid $\mathrm{NH}_{3}(30 \mathrm{ml})$ at $-78^{\circ} \mathrm{C}$, and $7 \mathrm{~h}(1.4 \mathrm{~g}, 3.97 \mathrm{mmol})$ was added. The mixture was stirred for $10 \mathrm{~min}$, then methyl iodide $(0.25 \mathrm{ml}, 3.97 \mathrm{mmol})$ was added. The reaction mixture was allowed to warm to room temperature, and stirred for $1 \mathrm{~h}$. It was evaporated, and the resultant residue was dissolved in ether. This solution was washed with water and brine, dried over $\mathrm{MgSO}_{4}$, and evaporated in vacuo. The residue was purified by column chromatography on silica gel with $n$-hexane-ethyl acetate $(30: 1)$ to give $7 \mathbf{i}(0.9 \mathrm{~g}, 61.9 \%)$ as a colorless oil. IR (Nujol): $1200 \mathrm{~cm}^{-1} .{ }^{1} \mathrm{H}-\mathrm{NMR}$ $\left(\mathrm{CDCl}_{3}\right) \delta: 1.89(3 \mathrm{H}, \mathrm{s}), 2.78-2.95(4 \mathrm{H}, \mathrm{m}), 3.06(3 \mathrm{H}, \mathrm{s}), 3.32(1 \mathrm{H}, \mathrm{d}$, $J=14 \mathrm{~Hz}), 3.58(1 \mathrm{H}, \mathrm{d}, J=14 \mathrm{~Hz}), 7.03-7.30(10 \mathrm{H}, \mathrm{m}), 7.40-7.58(4 \mathrm{H}$, $\mathrm{m})$.

4-Methyl-4-phenyl-4,5,6,7-tetrahydrothieno[3,2-c]pyridine Hydrochlo- 
ride (2a); Method A A mixture of $7 \mathbf{a}(0.72 \mathrm{~g}, 2.25 \mathrm{mmol})$ and $10 \% \mathrm{Pd}-\mathrm{C}$ $(0.7 \mathrm{~g})$ in acetic acid $(25 \mathrm{ml})$ was hydrogenated with $\mathrm{H}_{2}(30 \mathrm{~atm})$ at $80^{\circ} \mathrm{C}$ for $6 \mathrm{~h}$. The catalyst was removed by filtration, and the filtrate was evaporated in vacuo. The residue was dissolved in ethyl acetate. The solution was washed with saturated aqueous $\mathrm{NaHCO}_{3}$ and brine, dried over $\mathrm{MgSO}_{4}$, and evaporated in vacuo. The residue was dissolved in $6 \mathrm{~N}$ $\mathrm{HCl}$ in ethanol, and the solution was cooled to $0^{\circ} \mathrm{C}$. The precipitates were recrystallized from a mixture of ethanol and ether $(1: 1)$ to give $\mathbf{2 a}$ in $6.0 \%$ yield $(0.04 \mathrm{~g}, 0.15 \mathrm{mmol})$ as a white solid. The physical properties and spectral data for this compound are listed in Tables 2 and 4 Compounds $\mathbf{2 k}, \mathbf{3 f}$, and $\mathbf{4 b}$, c were similarly prepared from $7 \mathbf{c}, \mathbf{g}-\mathbf{i}$ respectively, using catalytic hydrogenation at $1 \mathrm{~atm}$. Their physical properties and spectral data are listed in Tables 2 and 4.

Method B $\left(\mathrm{NH}_{4}\right)_{2} \mathrm{Ce}\left(\mathrm{NO}_{3}\right)_{6}(4.70 \mathrm{~g}, 8.58 \mathrm{mmol})$ was added to a suspension of $7 \mathbf{b}(1.0 \mathrm{~g}, 2.86 \mathrm{mmol})$ in $\mathrm{CH}_{3} \mathrm{CN}(18 \mathrm{ml})$ and $\mathrm{H}_{2} \mathrm{O}(9 \mathrm{ml})$, and the mixture was stirred at room temperature for $7 \mathrm{~h}$. Methanol $(5 \mathrm{ml})$ and $4 \mathrm{~N}$ aqueous $\mathrm{NaOH}(5 \mathrm{ml})$ were added, and the resultant mixture was poured into water $(50 \mathrm{ml})$, and extracted with $\mathrm{CH}_{2} \mathrm{Cl}_{2}(50 \mathrm{ml})$. The extract was washed with brine, dried over $\mathrm{MgSO}_{4}$, and evaporated in vacuo. The residue was dissolved in $6 \mathrm{~N} \mathrm{HCl}$ in ethanol, and the solution was cooled to $0^{\circ} \mathrm{C}$. The precipitates were recrystallized from a mixture of ethanol and ether $(1: 1)$ to give $2 \mathrm{a}$ in $19.7 \%$ yield $(0.15 \mathrm{~g}, 0.56 \mathrm{mmol})$. Compounds $\mathbf{2 c}$, and $\mathbf{3 a}$, d, c were similarly prepared from $\mathbf{7 b}, \mathbf{d}-\mathbf{f}$, respectively. Spectral data for these compounds are listed in Tables 2 and 4.

Method $\mathrm{C} \mathrm{BF}_{3}$-etherate $(0.52 \mathrm{ml}, 4.22 \mathrm{mmol})$ was added to a solution of $5 \mathrm{a}(1.0 \mathrm{~g}, 4.69 \mathrm{mmol})$ in tetrahydrofuran (THF) $(10 \mathrm{ml})$ at $-5^{\circ} \mathrm{C}$, and the solution was stirred for an additional $30 \mathrm{~min}$, then cooled to $-70^{\circ} \mathrm{C}$. Then $1.06 \mathrm{~m}$ methyllithium in $n$-hexane $(7.0 \mathrm{ml})$ was added. The reaction mixture was stirred for $1 \mathrm{~h}$ at the same temperature, saturated aqueous $\mathrm{NH}_{4} \mathrm{Cl}$ was added, and the whole was extracted with ethyl acetate. The extract was washed with water and brine, dried over $\mathrm{MgSO}_{4}$, and evaporated in vacuo. The residue was dissolved in $6 \mathrm{~N} \mathrm{HCl}$ in ethanol, and the solution was cooled to $0{ }^{\circ} \mathrm{C}$. The precipitates were recrystallized from a mixture of ethanol and ether $(1: 1)$ to give $2 \mathbf{a}$ in $15.2 \%$ yield $(0.19 \mathrm{~g}, 0.71 \mathrm{mmol})$. Compounds $\mathbf{2 f}$, i were similarly prepared. Spectral

Table 4. Spectral Data for Compounds $2-4$ data for these compounds are listed in Tables 2 and 4.

Method D. 4-Phenyl-6,7-dihydrothieno[3,2-c]pyridine $\mathrm{N}$-Oxide (8a) $m$-Chloroperbenzoic acid $(2.02 \mathrm{~g}, 9.38 \mathrm{mmol})$ was added to a solution of $5 \mathrm{a}(2.0 \mathrm{~g}, 9.38 \mathrm{mmol})$ in $\mathrm{CH}_{2} \mathrm{Cl}_{2}(20 \mathrm{ml})$ at $0^{\circ} \mathrm{C}$. The reaction mixture was stirred for $1 \mathrm{~h}$ at room temperature, and poured into saturated aqueous $\mathrm{Na}_{2} \mathrm{~S}_{2} \mathrm{O}_{3}$. The organic layer was separated, washed with saturated aqueous $\mathrm{NaHCO}_{3}$ and brine, dried over $\mathrm{MgSO}_{4}$, and evaporated in vacuo to give $8 \mathbf{a}(65.4 \%, 1.50 \mathrm{~g})$ as a pale yellow solid, $\mathrm{mp}$ 98 - $99^{\circ} \mathrm{C}$. IR (Nujol): $1615,1545,1520 \mathrm{~cm}^{-1} .{ }^{1} \mathrm{H}-\mathrm{NMR}\left(\mathrm{CDCl}_{3}\right) \delta$ : $3.26-3.34(2 \mathrm{H}, \mathrm{m}), 4.36-4.44(2 \mathrm{H}, \mathrm{m}), 6.69(1 \mathrm{H}, \mathrm{d}, J=5.2 \mathrm{~Hz}), 7.10$ $(1 \mathrm{H}, \mathrm{d}, J=5.2 \mathrm{~Hz}), 7.37-7.51(3 \mathrm{H}, \mathrm{m}), 7.65-7.72(2 \mathrm{H}, \mathrm{m}) . \mathrm{MS} m / z$ : $230\left(\mathrm{M}^{+}+1\right)$. Anal. Calcd for $\mathrm{C}_{13} \mathrm{H}_{11}$ NOS: C, $68.10 ; \mathrm{H}, 4.84 ; \mathrm{N}, 6.11$. Found: $\mathrm{C}, 67.92 ; \mathrm{H}, 4.88 ; \mathrm{N}, 6.10$. The following compound was prepared similarly, and was not further purified or analyzed before use in the next step.

4-(2-Thienyl)-6,7-dihydrothieno[3,2-c]pyridine $N$-Oxide (8b): 8 b was obtained from $\mathbf{5 c}$ in $49.1 \%$ yield as a pale yellow oil. IR (film): 1540 , $1500 \mathrm{~cm}^{-1} .{ }^{1} \mathrm{H}-\mathrm{NMR}\left(\mathrm{CDCl}_{3}\right) \delta: 3.22-3.33(2 \mathrm{H}, \mathrm{m}), 4.20-4.28(2 \mathrm{H}$, $\mathrm{m}), 7.24(1 \mathrm{H}, \mathrm{dd}, J=4.1,5.1 \mathrm{~Hz}), 7.52(1 \mathrm{H}, \mathrm{d}, J=5.3 \mathrm{~Hz}), 7.57(1 \mathrm{H}, \mathrm{d}$, $J=5.3 \mathrm{~Hz}), 7.66(1 \mathrm{H}, \mathrm{d}, J=5.1 \mathrm{~Hz}), 7.84(1 \mathrm{H}, \mathrm{d}, J=4.1 \mathrm{~Hz})$.

4-Phenyl-6,7-dihydrothieno[2,3-c]pyridine $N$-oxide (8c) and 1-phenyl3,4-dihydro[1] benzothieno[3,2-c]pyridine $N$-oxide (8d) were not isolated before use in the next step.

$N$-Hydroxy-4-methyl-4-phenyl-4,5,6,7-tetrahydrothieno[3,2-c]pyridine (9a) Methylmagnesium bromide $(4.25 \mathrm{mmol})$ in ether $(1.42 \mathrm{ml})$ was added to a solution of $8 \mathbf{a}(0.75 \mathrm{~g}, 3.27 \mathrm{mmol})$ in THF $(10 \mathrm{ml})$ at room temperature. The reaction mixture was stirred for $1 \mathrm{~h}$, poured into saturated aqueous $\mathrm{NH}_{4} \mathrm{Cl}(20 \mathrm{ml})$ at $0{ }^{\circ} \mathrm{C}$, and then extracted with ethyl acetate. The extract was washed with brine, dried over $\mathrm{MgSO}_{4}$, and evaporated in vacuo. The residue was recrystallized from ether to give 9a $(84.8 \%, 0.68 \mathrm{~g})$ as a pale yellow solid, $\mathrm{mp} 174-176^{\circ} \mathrm{C}$. IR (Nujol): $3200,3180,1495 \mathrm{~cm}^{-1} .{ }^{1} \mathrm{H}-\mathrm{NMR}\left(\mathrm{CDCl}_{3}\right) \delta: 1.86(3 \mathrm{H}, \mathrm{s}), 2.77-2.88$ $(1 \mathrm{H}, \mathrm{m}), 2.97-3.26(3 \mathrm{H}, \mathrm{m}), 6.59(1 \mathrm{H}, \mathrm{br}), 6.66(1 \mathrm{H}, \mathrm{d}, J=5.2 \mathrm{~Hz}), 7.10$ $(1 \mathrm{H}, \mathrm{d}, J=5.2 \mathrm{~Hz}), 7.17-7.31(5 \mathrm{H}, \mathrm{m})$. MS $m / z: 246\left(\mathrm{M}^{+}+1\right)$. Anal. Calcd for $\mathrm{C}_{14} \mathrm{H}_{15} \mathrm{NOS} \cdot 0.1 \mathrm{H}_{2} \mathrm{O}: \mathrm{C}, 68.04 ; \mathrm{H}, 6.20 ; \mathrm{N}, 5.67$. Found: $\mathrm{C}$, $68.16 ; \mathrm{H}, 6.53 ; \mathrm{N}, 5.60$. The following compounds were prepared

\begin{tabular}{|c|c|c|}
\hline $\begin{array}{l}\text { Compd. } \\
\text { No. }\end{array}$ & $\begin{array}{l}\text { IR (Nujol) } \\
\mathrm{cm}^{-1}\end{array}$ & ${ }^{1} \mathrm{H}-\mathrm{NMR}(\mathrm{ppm})^{a)}$ \\
\hline $2 \mathbf{a}$ & 1580 & $\begin{array}{l}2.06(3 \mathrm{H}, \mathrm{s}), 2.90-3.49(4 \mathrm{H}, \mathrm{m}), 6.93(1 \mathrm{H}, \mathrm{d}, J=5.3 \mathrm{~Hz}), 7.32-7.48(5 \mathrm{H}, \mathrm{m}), 7.53(1 \mathrm{H}, \mathrm{d}, J=5.3 \mathrm{~Hz}), 9.92(1 \mathrm{H}, \\
\mathrm{br}), 10.46(1 \mathrm{H}, \mathrm{br})\end{array}$ \\
\hline $2 \mathbf{c}$ & 1580 & $\begin{array}{l}0.95(3 \mathrm{H}, \mathrm{t}, J=7.4 \mathrm{~Hz}), 2.35-2.57(2 \mathrm{H}, \mathrm{m}), 2.97-3.06(2 \mathrm{H}, \mathrm{m}), 3.20-3.46(2 \mathrm{H}, \mathrm{m}), 7.06(1 \mathrm{H}, \mathrm{d}, J=5.3 \mathrm{~Hz}) \\
7.39-7.41(5 \mathrm{H}, \mathrm{m}), 7.57(1 \mathrm{H}, \mathrm{d}, J=5.3 \mathrm{~Hz}), 9.96(1 \mathrm{H}, \mathrm{br}), 10.12(1 \mathrm{H}, \mathrm{br})\end{array}$ \\
\hline $2 d$ & 1560 & $2.07(3 \mathrm{H}, \mathrm{s}), 2.77(3 \mathrm{H}, \mathrm{s}), 3.25-3.67(4 \mathrm{H}, \mathrm{m}), 6.54(1 \mathrm{H}, \mathrm{d}, J=5 \mathrm{~Hz}), 7.32-7.58(6 \mathrm{H}, \mathrm{m})$ \\
\hline $2 \mathrm{e}$ & 1560 & $\begin{array}{l}1.23,1.37 \text { (total } 3 \mathrm{H}, \mathrm{t}, J=7 \mathrm{~Hz}), 2.07,2.22(\text { total } 3 \mathrm{H}, \mathrm{s}), 3.12-3.55(4 \mathrm{H}, \mathrm{m}), 3.73,3.78 \text { (total } 2 \mathrm{H}, \mathrm{q}, J=7 \mathrm{~Hz}), 6.40 \text {, } \\
6.61(\text { total } 1 \mathrm{H}, \mathrm{d}, J=5 \mathrm{~Hz}), 7.28-7.55(6 \mathrm{H}, \mathrm{m})\end{array}$ \\
\hline 2f & 1585 & $\begin{array}{l}1.57(3 \mathrm{H}, \mathrm{s}), 2.95-3.36(6 \mathrm{H}, \mathrm{m}), 6.86(1 \mathrm{H}, \mathrm{d}, J=5.3 \mathrm{~Hz}), 7.24-7.38(5 \mathrm{H}, \mathrm{m}), 7.42(1 \mathrm{H}, \mathrm{d}, J=5.3 \mathrm{~Hz}), 9.34(1 \mathrm{H} \\
\mathrm{br}), 10.13(1 \mathrm{H}, \mathrm{br})\end{array}$ \\
\hline $2 \mathrm{~g}$ & 1580 & $\begin{array}{l}2.13(3 \mathrm{H}, \mathrm{s}), 3.07-3.35(4 \mathrm{H}, \mathrm{m}), 7.00(1 \mathrm{H}, \mathrm{d}, J=6 \mathrm{~Hz}), 7.09(1 \mathrm{H}, \mathrm{dd}, J=4,6 \mathrm{~Hz}), 7.18(1 \mathrm{H}, \mathrm{d}, J=4 \mathrm{~Hz}), 7.47(1 \mathrm{H}, \\
\mathrm{d}, J=6 \mathrm{~Hz}), 7.56(1 \mathrm{H}, \mathrm{d}, J=6 \mathrm{~Hz})\end{array}$ \\
\hline $2 \mathbf{h}$ & 1560 & $\begin{array}{l}2.25(3 \mathrm{H}, \mathrm{s}), 2.56,2.58(\text { total } 3 \mathrm{H}, \mathrm{s}), 3.27-3.56(4 \mathrm{H}, \mathrm{m}), 6.90(1 \mathrm{H}, \mathrm{d}, J=5.3 \mathrm{~Hz}), 7.16(1 \mathrm{H}, \mathrm{dd}, J=2.6,5.2 \mathrm{~Hz}), 7.28 \\
(1 \mathrm{H}, \mathrm{d}, J=2.6 \mathrm{~Hz}), 7.46(1 \mathrm{H}, \mathrm{d}, J=5.3 \mathrm{~Hz}), 7.72(1 \mathrm{H}, \mathrm{d}, J=5.2 \mathrm{~Hz})\end{array}$ \\
\hline $2 \mathbf{i}$ & 1575 & $\begin{array}{l}2.03(3 \mathrm{H}, \mathrm{s}), 3.03-3.49(4 \mathrm{H}, \mathrm{m}), 6.97(1 \mathrm{H}, \mathrm{d}, J=5.3 \mathrm{~Hz}), 7.24(1 \mathrm{H}, \mathrm{d}, J=5.1 \mathrm{~Hz}), 7.26(1 \mathrm{H}, \mathrm{s}), 7.51(1 \mathrm{H}, \mathrm{d} \\
J=5.3 \mathrm{~Hz}), 7.61(1 \mathrm{H}, \mathrm{d}, J=5.1 \mathrm{~Hz})\end{array}$ \\
\hline $2 \mathbf{j}$ & 1580 & 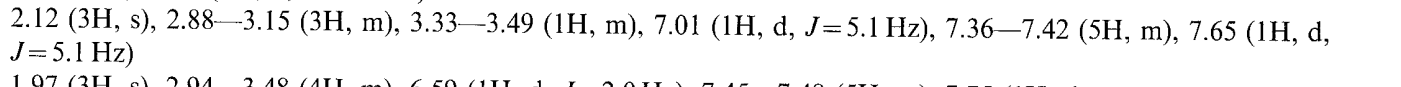 \\
\hline $2 \mathbf{k}$ & 1580 & $\begin{array}{l}1.97(3 \mathrm{H}, \mathrm{s}), 2.94-3.48(4 \mathrm{H}, \mathrm{m}), 6.59(1 \mathrm{H}, \mathrm{d}, J=2.0 \mathrm{~Hz}), 7.45-7.48(5 \mathrm{H}, \mathrm{m}), 7.75(1 \mathrm{H}, \mathrm{d}, J=2.0 \mathrm{~Hz}), 7.44-7.53 \\
(4 \mathrm{H}, \mathrm{m}), 10.12(1 \mathrm{H}, \mathrm{br}), 10.60(1 \mathrm{H}, \mathrm{br})\end{array}$ \\
\hline 3a & 1580 & $\begin{array}{l}2.21(3 \mathrm{H}, \mathrm{s}), 2.96-3.45(4 \mathrm{H}, \mathrm{m}), 7.02(1 \mathrm{H}, \mathrm{dd}, J=4,5 \mathrm{~Hz}), 7.17(1 \mathrm{H}, \mathrm{d}, J=4 \mathrm{~Hz}), 7.26-7.33(5 \mathrm{H}, \mathrm{m}), 7.55(1 \mathrm{H}, \mathrm{d}, \\
J=5 \mathrm{~Hz})\end{array}$ \\
\hline $3 \mathbf{b}$ & 2320,1260 & $2.27(3 \mathrm{H}, \mathrm{s}), 2.89(3 \mathrm{H}, \mathrm{s}), 3.25-3.74(4 \mathrm{H}, \mathrm{m}), 7.08-7.29(4 \mathrm{H}, \mathrm{m}), 7.33-7.48(2 \mathrm{H}, \mathrm{m}), 7.60(1 \mathrm{H}, \mathrm{d}, J=5 \mathrm{~Hz})$ \\
\hline $3 \mathbf{c}$ & 2380,1280 & $1.34(3 \mathrm{H}, \mathrm{t}, J=7 \mathrm{~Hz}), 2.10-2.35(2 \mathrm{H}, \mathrm{m}), 3.25-3.80(4 \mathrm{H}, \mathrm{m}), 7.08-7.40(6 \mathrm{H}, \mathrm{m}), 7.60(1 \mathrm{H}, \mathrm{d}, J=5 \mathrm{~Hz})$ \\
\hline 3d & 1600,1580 & $\begin{array}{l}2.16(3 \mathrm{H}, \mathrm{s}), 2.22(3 \mathrm{H}, \mathrm{s}), 2.86-3.38(4 \mathrm{H}, \mathrm{m}), 3.77(3 \mathrm{H}, \mathrm{m}), 6.77-6.88(2 \mathrm{H}, \mathrm{m}), 7.03(1 \mathrm{H}, \mathrm{dd}, J=4,5 \mathrm{~Hz}), 7.19 \\
(1 \mathrm{H}, \mathrm{s}), 7.25(1 \mathrm{H}, \mathrm{d}, J=7 \mathrm{~Hz}), 7.54(1 \mathrm{H}, \mathrm{d}, J=5 \mathrm{~Hz})\end{array}$ \\
\hline $3 \mathbf{e}$ & 1580 & $2.12(3 \mathrm{H}, \mathrm{s}), 2.80-3.45(4 \mathrm{H}, \mathrm{m}), 7.15-7.28(5 \mathrm{H}, \mathrm{m}), 7.52-7.62(1 \mathrm{H}, \mathrm{m})$ \\
\hline $3 f$ & 1580 & $2.10(3 \mathrm{H}, \mathrm{s}), 3.12-3.56(4 \mathrm{H}, \mathrm{m}), 6.43-6.60(3 \mathrm{H}, \mathrm{m}), 7.20-7.40(6 \mathrm{H}, \mathrm{m}), 7.73-7.80(1 \mathrm{H}, \mathrm{m})$ \\
\hline $4 \mathbf{a}$ & 1570 & $2.20(3 \mathrm{H}, \mathrm{s}), 3.45-3.54(4 \mathrm{H}, \mathrm{m}), 7.43-7.53(7 \mathrm{H}, \mathrm{m}), 7.82(1 \mathrm{H}, \mathrm{d}, J=7 \mathrm{~Hz}), 8.01(1 \mathrm{H}, \mathrm{d}, J=7 \mathrm{~Hz})$ \\
\hline $4 \mathbf{b}$ & 3250,1575 & $2.20(3 \mathrm{H}, \mathrm{s}), 2.64-3.55(4 \mathrm{H}, \mathrm{m}), 7.02-7.63(9 \mathrm{H}, \mathrm{m}), 9.98(1 \mathrm{H}, \mathrm{br})$ \\
\hline $4 c$ & 1570 & $2.27(3 \mathrm{H}, \mathrm{s}), 3.00-3.18(2 \mathrm{H}, \mathrm{m}), 3.23(3 \mathrm{H}, \mathrm{s}), 3.30-3.55(2 \mathrm{H}, \mathrm{m}), 6.99-7.23(3 \mathrm{H}, \mathrm{m}), 7.25-7.60(6 \mathrm{H}, \mathrm{m})$ \\
\hline
\end{tabular}

a) Listed as chemical shifts (number of protons, multiplicity, constant). All compounds were dissolved in DMSO- $d_{6}$. 
similarly; 9d was not further purified or analyzed before use in the next step.

$N$-Hydroxy-4-methyl-4-(2-thienyl)-4,5,6,7-tetrahydrothieno[3,2-c]pyridine (9b): $9 \mathbf{b}$ was obtained from $\mathbf{8 b}$ in $85.5 \%$ yield as a pale yellow solid, $171-173{ }^{\circ} \mathrm{C}(\mathrm{EtOH})$. IR (Nujol): $3070 \mathrm{~cm}^{-1} .{ }^{1} \mathrm{H}-\mathrm{NMR}$ (DMSO$\left.d_{6}\right) \delta: 1.73(3 \mathrm{H}, \mathrm{s}), 2.40-3.10(4 \mathrm{H}, \mathrm{m}), 6.42(1 \mathrm{H}, \mathrm{d}, J=4 \mathrm{~Hz}), 6.67-6.78$ $(2 \mathrm{H}, \mathrm{m}), 7.16(1 \mathrm{H}, \mathrm{d}, J=5 \mathrm{~Hz}), 7.24(1 \mathrm{H}, \mathrm{d}, J=5 \mathrm{~Hz}), 8.03(1 \mathrm{H}, \mathrm{s}) . \mathrm{MS}$ $m / z: 251\left(\mathrm{M}^{+}\right)$. Anal. Calcd for $\mathrm{C}_{12} \mathrm{H}_{13} \mathrm{NOS}_{2}: \mathrm{C}, 57.34 ; \mathrm{H}, 5.21 ; \mathrm{N}, 5.57$. Found: C, 57.08; H, 5.13; N, 5.46.

$N$-Hydroxy-4-methyl-4-phenyl-4,5,6,7-tetrahydrothieno[2,3-c]pyridine (9c): $9 \mathbf{c}$ was obtained from $8 c$ in $47.8 \%$ yield $(0.55 \mathrm{~g})$ as a pale yellow solid, $177-178^{\circ} \mathrm{C}\left(\mathrm{Et}_{2} \mathrm{O}\right)$. IR (Nujol): $3250,1490 \mathrm{~cm}^{-1} .{ }^{1} \mathrm{H}-\mathrm{NMR}$ $\left(\mathrm{CDCl}_{3}\right) \delta: 1.97(3 \mathrm{H}, \mathrm{s}), 2.57-2.65(1 \mathrm{H}, \mathrm{m}), 3.00-3.35(3 \mathrm{H}, \mathrm{m}), 6.82$ (1H, br), $6.83(1 \mathrm{H}, \mathrm{d}, J=5.1 \mathrm{~Hz}), 7.21-7.29(6 \mathrm{H}, \mathrm{m})$. MS $m / z: 245\left(\mathrm{M}^{+}\right)$. Anal. Calcd for $\mathrm{C}_{14} \mathrm{H}_{15} \mathrm{NOS} \cdot 0.1 \mathrm{H}_{2} \mathrm{O}: \mathrm{C}, 68.03 ; \mathrm{H}, 6.19 ; \mathrm{N}, 5.66$. Found: C, $68.14 ; \mathrm{H}, 6.11 ; \mathrm{N}, 5.63$.

$N$-Hydroxy-1-methyl-1-phenyl-1,2,3,4-tetrahydro[1]benzothieno[2,3c]pyridine (9d): $9 \mathbf{d}$ was obtained from $8 \mathbf{d}$ in $44.5 \%$ yield $(0.75 \mathrm{~g})$ as a pale yellow solid, $188-189^{\circ} \mathrm{C}\left(\mathrm{Et}_{2} \mathrm{O}\right)$. IR (Nujol): $3230,1495 \mathrm{~cm}^{-1}$. ${ }^{1} \mathrm{H}-\mathrm{NMR}\left(\mathrm{CDCl}_{3}\right) \delta: 1.96(3 \mathrm{H}, \mathrm{s}), 2.58-3.42(4 \mathrm{H}, \mathrm{m}), 7.14-7.38(7 \mathrm{H}$, m), $7.49-7.88(2 \mathrm{H}, \mathrm{m})$. MS $m / z: 295\left(\mathrm{M}^{+}\right)$.

2a (Method D) $\mathrm{PCl}_{3}(0.2 \mathrm{ml}, 2.3 \mathrm{mmol})$ was added to a solution of 9a $(0.55 \mathrm{~g}, 2.24 \mathrm{mmol})$ in THF $(5 \mathrm{ml})$ at $0^{\circ} \mathrm{C}$, and the reaction mixture was stirred for $1 \mathrm{~h}$ at room temperature, then poured into water. The whole was adjusted to $\mathrm{pH} 9$ with saturated aqueous $\mathrm{K}_{2} \mathrm{CO}_{3}$, and extracted with ethyl acetate. The extract was washed with water and brine, dried over $\mathrm{MgSO}_{4}$, and evaporated in vacuo. The residue was dissolved in $6 \mathrm{~N}$ $\mathrm{HCl}$ in ethanol, and the whole was cooled to $0^{\circ} \mathrm{C}$. The precipitates were recrystallized from a mixture of ethanol and ether $(1: 1)$ to give $2 \mathrm{a}$ in $50.4 \%$ yield $(0.30 \mathrm{~g}, 1.13 \mathrm{mmol})$. Compounds $2 \mathrm{~g}$, $\mathbf{j}$ and $4 \mathbf{a}$ were prepared similarly. Physical properties and spectral data for these compounds are listed in Tables 2 and 4.

$(+)$ and (-)-4-Methyl-4-phenyl-4,5,6,7-tetrahydrothieno[3,2-c]pyridine Hydrochloride $((+)-2 \mathrm{a}$ and $(-)-2 \mathrm{a}) \quad \mathrm{A}$ solution of $(+)$-di- $p$ toluoyl-D-tartaric acid $(1.3 \mathrm{~g}, 8.72 \mathrm{mmol})$ in $\mathrm{EtOH}(12 \mathrm{ml})$ was added to a solution of salt-free $2 \mathrm{a}(2.0 \mathrm{~g}, 8.72 \mathrm{mmol})$ in EtOH $(20 \mathrm{ml})$, and the whole was allowed to stand for $5 \mathrm{~d}$. The crystals were collected by filtration, then suspended in a mixture of ethyl acetate and water. The mixture was adjusted to $\mathrm{pH} 10$ with saturated aqueous $\mathrm{K}_{2} \mathrm{CO}_{3}$. The organic layer was separated, and washed with water and brine, dried over $\mathrm{MgSO}_{4}$, and evaporated in vacuo. The residue was dissolved in $\mathrm{EtOH}(5 \mathrm{ml})$, and $6 \mathrm{~N} \mathrm{HCl} \mathrm{in} \mathrm{EtOH}(1 \mathrm{ml})$ was added at $0^{\circ} \mathrm{C}$. The resulting white crystals were collected by filtration and washed with ether to give (+)-2a (360 mg, 18.0\%). Similarly, (-)-2a was obtained from salt-free 2a $(5.1 \mathrm{~g}, 22.2 \mathrm{mmol})$ in $23.4 \%$ yield using (-)-di-p-toluoyl-D-tartaric acid as the resolving agent. Resolution of $\mathbf{2} \mathbf{g}$ and $\mathbf{3 a}$ was performed in the same way. Physical properties and spectral data for these optical isomers are listed in Tables 3 and 4.

Biological Activities. Anticonvulsant Activity against NMDA-Induced Seizure in Mice Five ICR male mice of the same age were used per group. One group of mice was treated i.p. with a test compound dissolved in saline, and the other group was given saline alone, $30 \mathrm{~min}$ before the experiment. NMDA $(0.1$ or $0.32 \mu \mathrm{g})$ dissolved in saline $(0.5 \mu \mathrm{l})$ was injected i.c.v. into each mouse. Each mouse was then placed in a plastic cage, and observed for $10 \mathrm{~min}$ to confirm the occurrence of clonic and tonic seizures. The latencies of initial seizures (seconds) were compared using the Mann-Whitney $U$-test.

Anti-hypoxia $\left(\mathbf{9 8 \%} \mathrm{N}_{2}-\mathbf{2 \%} \mathrm{O}_{2}\right)$ Activity in Mice Mice were main-
Table 5. Crystal Data for (-)-3a (+)-Di-p-toluoyl-D-tartarate

\begin{tabular}{|c|c|}
\hline Formula & $\mathrm{C}_{14} \mathrm{H}_{13} \mathrm{NS}_{2} \cdot \mathrm{C}_{20} \mathrm{H}_{18} \mathrm{O}_{8}$ \\
\hline Formula weight & 645.74 \\
\hline Crystal color, habit & Colorless, prismatic \\
\hline Crystal dimensions (mm) & $0.25 \times 0.20 \times 0.20$ \\
\hline Crystal system & Monoclinic \\
\hline Space group & $P 2_{1}$ \\
\hline Lattice parameters: $a, \AA$ & 7.887 \\
\hline$b, \AA$ & 12.018 \\
\hline$c, \AA$ & 16.397 \\
\hline$\beta$, degree & 92.90 \\
\hline$V, \AA^{3}$ & 1552 \\
\hline$Z$ value & 2 \\
\hline Density (calcd), $\mathrm{g} / \mathrm{cm}^{3}$ & 1.382 \\
\hline
\end{tabular}

tained in a closed glass chamber in which a mixture of $98 \%$ nitrogen and $2 \%$ oxygen was circulated, and their survival time was measured. One group of mice was pretreated i.p. with a test compound dissolved in saline, and the other group was given saline alone, $30 \mathrm{~min}$ before the experiment. Five mice were used per group, and the mean values of the survival times were compared using the Mann-Whitney $U$-test. Minimum effective dose (MED) was defined as that showing a statistically significant effect $(p<0.05)$.

X-Ray Structure Determination Colorless prism crystals of (-)-3a $(+)$-di- $p$-toluoyl-D-tartarate were grown from ethanol. Diffraction measurements were performed on a Rigaku AFC-5R diffractometer using graphite-monochromated $\operatorname{MoK}_{\alpha}$ radiation $(\lambda=0.71069 \AA)$. Crystal data are shown in Table 5. A total of 4015 reflections ( 3751 unique reflections) were collected using the $\omega-2 \theta$ scan technique within a $2 \theta$ range of $55^{\circ}$. The structure was solved by a direct method and refined by a full-matrix least-squares methods using 2365 reflections $\left(I_{0}>2.5 \sigma_{\mathrm{I}}\right)$. The final refinement converged to $R=0.106$ and $R_{\mathrm{w}}=0.110$.

Acknowledgements The authors wish to thank the staff members of the Analytical Research Laboratories for elemental analysis and the measurement of NMR spectral data.

\section{References and Notes}

1) Ohkubo M., Kuno A., Katsuta K., Ueda Y., Shirakawa K., Nakanishi H., Nakanishi I., Imai K., Kinoshita T., Takasugi H., Chem. Pharm. Bull., 44, 95 (1996).

2) Mitrovic N., Besson M. J., Maurin Y., Eur. J. Pharmacol., 176, 357 (1990).

3) Herz W., J. Am. Chem. Soc., 73, 351 (1951).

4) Bremner J. B., Browne E. J., Chohan V., Yates B. F., Aust. J. Chem., 37, 1043 (1984).

5) Kobayashi M., Kitazawa M., Saito T., Yamamoto R., Harada H., Japan Kokai Tokkyo Koho, JP60152483 [Chem. Abstr., 103, 215153 (1985)].

6) Foye W. O., Speranza J. P., Eur. J. Med. Chem., 9, 177 (1974).

7) Gray N. M., Cheng B. K., Mick S. J., Lair C. M., Contreras P. C., J. Med. Chem., 32, 1242 (1989).

8) Herz W., J. Am. Chem. Soc., 72, 4999 (1950).

9) Kiguchi T., Kuninobu N., Takahashi Y., Yoshida Y., Naito T., Ninomiya I., Synthesis, 1989, 778. 